\title{
Kampf der Kulturbegriffe? Eine Fallstudie zum wissenschaftlichen Schreiben über „Kultur“ im BA-Studium „Transkulturelle Kommunikation"
}

\author{
Clash of Cultural Concepts? A case study on academic writing about \\ „culture" in the BA-Studies Transcultural Communication
}

\begin{abstract}
Der Umgang mit dem Begriff „Kultur“ und den Konzepten, auf die sich der Begriff bezieht, stellt hohe heuristische und rhetorische Anforderungen an Studierende der Translationswissenschaft. „Kultur“ wird im Fachdiskurs auf unterschiedliche, teilweise widersprüchliche Konzepte bezogen. In Seminararbeiten fällt es Studierenden oft schwer, sich nachhaltig von homogenisierenden, nationalisierenden Kulturkonzepten zu emanzipieren, selbst wenn der thematische Fokus der Arbeit genau dies nahelegen würde.

In meinem Beitrag werde ich dieses Phänomen anhand der ersten Fassung einer BA-Arbeit aus dem Seminar Transkulturelle Kommunikation (WS 2017/18) analysieren. Dabei wird das Problem des Umgangs mit dem Begriff „Kultur“ aus verschiedenen Perspektiven beleuchtet werden: Einerseits werden Anforderungen und Herausforderungen im Umgang mit dem Begriff „Kultur“ auf einer inhaltlich-begrifflichen Ebene analysiert, andererseits im Hinblick auf die Schreibentwicklung im wissenschaftlichen Schreiben im Studium.

Die Analyse zeigt, wie durch einen undifferenzierten Umgang mit dem Begriff „Kultur“ Vorannahmen in die BAArbeit hineingetragen werden, die einerseits in der theoretischen Auseinandersetzung mit dem Thema und andererseits vor allem auch in der Entwicklung eines Fragebogens zu gravierenden Problemen führen. Es wird argumentiert, dass es demnach eine wichtige Aufgabe der Translationsdidaktik ist, Studierende in der Aneignung differenzierter Kulturkonzepte zu unterstützen.
\end{abstract}

\section{Schlagworte}

Kulturkonzepte; Transkulturelle Kommunikation; Wissenschaftliches Schreiben; Translationsdidaktik; Wissensverarbeitung; Textanalyse

\section{Zum Gegenstand: Schreiben über „Kultur“ in der Translationswissenschaft}

„Kultur“ ist ein ebenso intensiv gebrauchter wie umstrittener Begriff. Die Debatte um den Terminus und vor allem seine Inhalte wird nicht nur wissenschaftlich geführt, sondern wirkt stark in das Alltagsverständnis hinein, hat gesellschaftliche und politische Konsequenzen und lässt in Social Media die Emotionen hochkochen. Eventuelle Kritik an Homogenisierung und Essentialisierung wird da - vor allem in Bezug auf den Migrationsdiskurs - zuweilen als ,ideologisch“ abgetan. Verkürzung und Vereinfachung in Bezug auf den Kulturbegriff führt zu gesellschaftspolitischen Problemen, legitimiert und befördert Diskriminierung und Ausgrenzung. Dies wurde und wird interdisziplinär viel diskutiert, und diese Diskussion ist noch lange nicht abgeschlossen.

\footnotetext{
* Sabine Dengscherz

Universität Wien

Zentrum für Translationswissenschaft

Gymnasiumstraße 50

1190 Wien

sabine.dengscherz@univie.ac.at
} 
In meinem Beitrag nähere ich mich der Debatte aus einer anderen Perspektive. Es geht mir um die Frage, wie sich Verkürzungen und Vereinfachungen rund um den Kulturbegriff auf das Erkenntnispotential beim wissenschaftlichen Arbeiten und Schreiben auswirken und dadurch die wissenschaftliche Argumentation erschweren. Dies soll exemplarisch anhand der Analyse einer BA-Arbeit gezeigt werden. Dabei geht es nicht darum, die Vorgehensweise des Studenten zu diskreditieren, sondern anhand eines authentischen Beispiels zu zeigen, was für Folgen das Ausgehen von nicht hinterfragten Vorannahmen für den Erkenntnisprozess haben kann. Die potentielle gesellschaftliche Relevanz der Analyse ergibt sich daraus, dass es sehr verbreitete Vorannahmen sind, von denen der Student hier in seiner Arbeit ausgeht: Vorannahmen, die mit einer Vernebelung des Kulturbegriffs einhergehen und die nicht nur im Alltagsdiskurs, sondern auch in Seminararbeiten immer wieder beobachtet werden können. Diese Vorannahmen wirken sich problematisch auf die Präzision - und zuweilen auch auf die Klarheit - der Argumentation im Text aus.

Klarheit und Präzision sind wichtige Qualitätskriterien für wissenschaftliche Texte. Es sollte möglichst eindeutig sein, worauf sich ein Begriff bezieht, und Konzepte müssen transparent gemacht und definiert werden. Es ist ein Zeichen von Expertise, wenn es gelingt, ein tiefes Verständnis von einem Gegenstand und seinen Dimensionen zu entwickeln und Wissen flexibel auf neue Kontexte anzuwenden (vgl. Bransford et al. 2000: 31). Dabei kollidieren zuweilen das Alltagsverständnis von einem Begriff und das wissenschaftliche Verständnis davon (bzw. unterschiedliche Versionen wissenschaftlichen Verständnisses von einem Begriff). Das Schreiben über „Kultur“ beziehungsweise die Verwendung des Begriffs „Kultur“ stellt hier besonders hohe Anforderungen an Studierende, zumal dabei auch unterschiedliche Diskurstraditionen berücksichtigt und Diskurspositionen in ihrer Historizität reflektiert werden müssen.

In meinem Beitrag setze ich mich mit heuristischen und rhetorischen Herausforderungen (vgl. Dengscherz 2017: 164 und 2018) beim Schreiben über „Kultur“ in der Translationswissenschaft auseinander. Ausgehend von einer BA-Arbeit aus dem Bereich der Transkulturellen BusinessKommunikation wird analysiert, auf welche Probleme der Schreiber stößt und wie er ihnen begegnet. Die Analyseergebnisse werden einerseits vor dem Hintergrund unterschiedlicher Kulturkonzepte im Fachdiskurs und andererseits vor dem Hintergrund der Schreibentwicklung im wissenschaftlichen Schreiben diskutiert.

Im Folgenden werden zunächst unterschiedliche Gegenstandsdimensionen behandelt, die als Hintergrund und theoretische Ausgangspunkte der Analyse zu betrachten sind: In Abschnitt 1.1. werden überblicksartig wichtige Unterscheidungslinien in Bezug auf Kulturkonzepte skizziert und in ihrer Relevanz für die Translationswissenschaft und die Transkulturelle Kommunikation reflektiert. In Abschnitt 1.2. geht es um für die Analyse relevante Modelle der akademischen Schreibentwicklung. Die darauffolgenden Abschnitte sind der Darstellung von Analysemethode und Datenbasis (2), der Präsentation (3) und Diskussion (4) der Analyseergebnisse sowie Schlussfolgerungen (5) und weiterführenden Überlegungen (6) gewidmet.

\subsection{Kulturkonzepte in der Transkulturellen Kommunikation}

Wie ,Kultur' konzeptionalisiert wird, spielt eine wichtige Rolle in der Transkulturellen Kommunikation. Wesentlich ist dabei die Unterscheidung von mindestens zwei Dimensionen des Kulturbegriffs, nämlich die inhaltliche Bedeutung von Kultur und ihre geographische oder nationale oder ethnische Extension (vgl. Welsch 2010: 39). In der inhaltlichen Dimension wird reflektiert, was Kultur eigentlich ist und was alles dazugehört, in der extensionalen Dimension wiederum, wer diese Kultur repräsentiert, wer ihr zuzuordnen ist und wo sie verbreitet ist. Die beiden Dimensionen werden nicht immer klar unterschieden. Im Alltagsverständnis wird „Kultur“ nicht selten auf eine Nation, Region, Religion oder Ethnie bezogen, wie Welsch (2010: 39) ausführt: „Wir denken bei ,Kultur' sogleich einen nationalen oder ethnischen Geltungsbereich mit, ja die extensionale Bedeutung hat meistens sogar die Führung vor der inhaltlichen Bedeutung". Welsch plädiert dafür, ,die erste, die inhaltliche, und die zweite, die extensionale Bedeutung von ,Kultur“ nicht wie selbstverständlich zu amalgamieren, sondern unterschieden zu halten.“ 
Die angesprochene Verknüpfung der inhaltlichen mit der extensionalen Bedeutung lässt sich auf den „herkömmlichen Kulturbegriff“ zurückführen, der sich gegen Ende des 18. Jahrhunderts herausgebildet und ,Kultur' auf zwei Ebenen, eng' gefasst hat (vgl. Saal 2014: 22): Auf der inhaltlichen Ebene wird die „sogenannte Hochkultur“ bezeichnet und auf der extensionalen Ebene eine homogenisiert gedachte Gruppe von Menschen. Es werden

\begin{abstract}
mit dem herkömmlichen Kulturbegriff - als Kulturen - Gruppen von Menschen bezeichnet, für die gemeinsame Wertvorstellungen und Denkmuster wie Sprache, Geschichte, Religion, ein ,kulturelles Erbe' (beispielsweise die o.g. Hochkultur in Europa) gelten und die einen geopolitischen Raum für sich in Anspruch nehmen; solche Gruppen gelten als in sich homogen. Mit dem herkömmlichen Kulturbegriff werden also sowohl alle weltanschaulichen, religiösen, ethnischen, künstlerischen, politischen und wissenschaftlichen Institutionen als auch eine solche Gemeinschaft selbst bezeichnet. (Saal 2014: 22)
\end{abstract}

Dieser herkömmliche Kulturbegriff, der häufig mit Herders Kugelmodell in Verbindung gebracht wird (vgl. Welsch 2010: 41 und zur Kritik einer vereinfachten Darstellung des Kugelmodells auch Saal 2014: 24-27), wurde nun im Zuge von Cultural Turns in den späten 1980er Jahren neuen, anderen Konzepten gegenübergestellt. „Kultur“ wird dabei sehr unterschiedlich konzeptionalisiert und reflektiert (vgl. Lutter/Reisenleitner 2002: 612). Den Pluralismus von Kulturkonzeptionen und den dahinterliegenden Forschungstraditionen macht Bachmann-Medick (2006) anschaulich, indem sie gleich von mehreren Cultural Turns spricht: Ausgehend von einem Linguistic Turn, der „Text und Repräsentation als Erkenntnisbedingung“ (Bachmann-Medick 2006: 34) versteht und damit Sprache und Kultur eng verknüpft, werden weitere Turns skizziert, die jeweils unterschiedliche Schwerpunkte setzen: Interpretative Turn (semiotischer Kulturbegriff, Kultur als Text), Performative Turn (Darstellung und performative Praxis, Kulturdynamik), Reflexive Turn/ Literary Turn (kritische Selbstreflexion der Ethnologie, v.a. ausgehend von der Literaturwissenschaft), Postcolonial Turn, (kritische Auseinandersetzung mit Identiät, Repräsentation und Hegemonie im - postkolonialen - internationalen Machtgefüge), Translational Turn (ein erweitertes Übersetzungsverständnis in der Translationswissenschaft wirkt in die Kulturwissenschaft: Kultur als „Übersetzung“), Spacial Turn (Tendenz zur Ortlosigkeit von Kultur, kritisches Re-Mapping) und Iconic Turn (Auseinandersetzung mit dem Erkenntniswert von Bildern, v.a. ausgehend von der Kunstwissenschaft). In unterschiedlichen Disziplinen werden also aus unterschiedlichen Perspektiven unterschiedliche Fragen aufgeworfen, Antworten gefunden beziehungsweise Entscheidungen getroffen, und so hat sich die Kulturreflexion durch Einflüsse aus diesen verschiedenen Disziplinen entwickelt und weiter ausdifferenziert. Als „Nährboden für die Herausbildung signifikanter cultural turns sowohl in den jeweiligen Einzeldisziplinen als auch quer zu ihnen"verortet Bachmann-Medick (2006: 9) die „Tendenz der Kulturwissenschaften zum Pluralismus, gepaart mit kritischer Selbstreflexion und mit (inter-)kultureller Verortung der eigenen Theorien“. Dies hat zur Ausbildung einer Reihe von unterschiedlichen Kultur-Konzepten geführt, die sich teilweise an inter-, multi- oder transkulturelle Ansätze andocken lassen. Die Neubewertung von „Kultur“ hat komplexe Aushandlungsprozesse ausgelöst, die immer noch im Gange sind.

Bei diesen Aushandlungsprozessen zu einer Neubewertung von „Kultur“ ging es auf der inhaltlichen Ebene in erster Linie um eine Erweiterung des Begriffs: Kultur bezieht sich nicht mehr nur auf ,Hochkultur', sondern auch auf ,Sprache und Text als Gestaltungs- und Triebkräfte sozialen Handelns" (Bachmann-Medick 2006: 13). Gerade die Erweiterung des Kulturbegriffs auf der inhaltlichen Ebene birgt nun umso mehr die Gefahren von Pauschalisierung und Essentialisierung - wenn dem nicht auch auf der extensionalen Ebene durch eine Neubewertung entgegengewirkt wird.

Ein solches Entgegenwirken haben Ansätze zum Ziel, die „gegen ein homogenisierendes und begrenzendes Kulturverständnis“ (Saal 2014: 21) gerichtet sind. Diese Ansätze, die die extensionale Dimension von „Kultur“ problematisieren, haben sich allerdings weniger flächendeckend durchgesetzt als die Erweiterung auf der inhaltlichen Ebene. Gerade die extensionale Ebene ist es auch, die für Studierende schwer zu fassen ist: Vorstellungen von kollektiven Identitäten sind 
hier häufig in einem „Zwischenreich aus Homogenität und Heterogenität“ (Hansen 2009: 160) angesiedelt, was dann beim wissenschaftlichen Arbeiten zu Inkonsistenzen und Verkürzungen im Umgang mit dem Kulturbegriff führen kann. Dies wird in der Analyse der BA-Arbeit noch zu sehen sein.

Für die Translationswissenschaft hat sich der Translational Turn, also die „kulturwissenschaftliche Neuorientierung der Übersetzungsforschung vor allem seit den späten 1980er Jahren“ (Bachmann-Medick 2006: 239) als äußerst fruchtbar erwiesen. Reiß/Vermeer verstehen Translation nicht nur als sprachlichen, sondern auch kulturellen Transfer (1984: 4) und Translator*innen dementsprechend nicht nur als Sprach-, sondern auch als „Kulturmittler“ (1984: 7). Sie betonen, „daß es bei einer Translation nicht um sprachliche, erst recht nicht um formale sprachliche Phänomene allein geht, daß Translation vielmehr ein kultureller und $\mathrm{d}$ a $\mathrm{r}$ i $\mathrm{n}$ sprachlicher Transferprozeß ist" (1984: 122). Texte werden also in ihrem Kontext, in ihrer Verwendungssituation betrachtet, es wird nicht allein text-immanent gearbeitet, sondern auch Außersprachliches einbezogen (vgl. Vermeer 2007: 114).

Sprache und Kultur als eng verbunden wahrzunehmen, die Sprache als „ein[en] wichtige[n] Träger und zugleich Ausdruck der jeweiligen Kultur“ (Kadrić et al. 2005: 26, vgl. dazu auch Kaiser-Cooke 2007) zu betrachten, schafft Erkenntnispotential, das nicht nur auf einer theoretischen Ebene Perspektiven eröffnet, sondern sich auch nutzbringend in die Praxis umsetzen lässt. Texte in ihrer Handlungsdimension zu begreifen und Translation damit in einer kommunikativen Situation zu verorten (wie dies etwa Justa Holz-Mänttäri 1984 in ihrer Theorie vom translatorischen Handeln tut, vgl. dazu auch Risku 1999: 108) schärft den Blick für die Verwendungssituation von Texten - und damit für außersprachliche Elemente der Kommunikation. Text ,in Kultur ', als Mittel zur sozialen Interaktion“ (Resch 1999: 165) zu begreifen, bedeutet, sich mit „,kulturspezifischen Konventionen“ (Resch 2012: 46) auseinanderzusetzen, nämlich damit, „wie in dieser Situation typischerweise getextet wird“ (ebd.). Dies ist wesentlich für die Professionalisierung translatorischen Handelns und eine der Grundlagen für ein funktionalistisches Textkonzept (vgl. Nord 1991: 264), in dem Translation als Textproduktion begriffen wird (vgl. Resch 2006). Über den Nutzen der inhaltlichen Erweiterung des Kulturbegriffs besteht weitgehend Konsens.

Schwieriger ist die extensionale Dimension zu definieren. Diese wird in der Translationswissenschaft oft als variables Element reflektiert: Ausgehend von konkreten Texten in konkreten Kommunikationssituationen lässt sich die Verortung von Ausgangs- und Zieltexten in ihrem jeweiligen kulturellen Kontext flexibel an die jeweiligen Kommunikationssituation anpassen: „Je nach Analysezweck kann demnach ,dasselbe‘ kulturelle Phänomen unterschiedlich eingeordnet werden" (vgl. Witte 2000: 56). Je nach Kommunikationssituation kann eine Firmenkultur, eine Domäne oder bestimmte (Wissenschafts-)Disziplin etc. mitgedacht werden - oder auch ein Faktorenbündel aus mehreren Aspekten.

Die Kollektive/Diskursgemeinschaften/Adressat*innen(gruppen), die in der translatorischen Kommunikationssituation berücksichtigt werden, können also auf sehr unterschiedlichen Ebenen gefasst und gedacht sein. ,Nationalkultur' als Kategorie hat damit zwar nicht völlig ausgedient, in den meisten Kommunikationssituationen greift diese Kategorie aber zu kurz, nicht zuletzt, weil dabei kollektive Verflechtungen unberücksichtigt bleiben, die die Kommunikationssituation wesentlich beeinflussen. Pöchhacker (1994) veranschaulicht das Problem mit der ,Nationalkultur anhand eines Beispiels aus dem internationalen Sport:

\footnotetext{
Man könnte praxisbezogen fragen, ob nicht etwa die schweizerischen, bundesdeutschen und die australischen Bob- und Rodelsportdelegierten beim Kongress der International Bobsleighing and Tobogganing Federation (Anchorage, 1989) gemeinsam eine Soziokultur konstituieren könnten, so dass von einer Art ,internationaler Diakultur“" zu sprechen wäre. (Pöchhacker 1994: 70)
}

Aber auch der Terminus ,Soziokultur' löst ,,freilich nicht das eigentliche begriffliche bzw. definitorische Problem. Im Gegenteil: Es ist zu fragen, wie der Begriff Soziokultur im einzelnen ,aufzufüllen" ist" (Pöchhacker 1994: 70). Das Problem liegt also nicht nur auf einer definitorischen Ebene, sondern vielmehr auf einer konzeptuellen. Pöchhacker verweist darauf, dass für die Lö- 
sung dieses Problems noch „Vorleistungen seitens der Soziologie oder der vergleichenden Kulturwissenschaft vonnöten“ (1994: 70) seien und plädiert damit für einen interdisziplinären Blick auf die Problematik.

In den 24 Jahren seit Pöchhackers Befund ist die interdisziplinäre Diskussion um die extensionale Dimension des Kulturbegriffs einige entscheidende Schritte weitergegangen. Dies hat allerdings weniger dazu geführt, dass auf einer definitorischen Ebene Klarheit gewonnen worden wäre, sondern vielmehr dazu, dass (vermeintliche) Sicherheiten noch weiter ins Wanken gebracht und als Verkürzungen entlarvt wurden. Pöchhacker hat 1994 dafür plädiert - vorübergehend, bis einige notwendige Klärungen vollzogen sind - von einem ,,integrativen und möglichst transparenten Kulturbegriff" auszugehen (S. 70). Dies erscheint zwar nach wie vor wünschenswert, allerdings in der Praxis - u.a. in der Translationsdidaktik - nicht immer leicht umzusetzen.

Wo die Knackpunkte liegen, zeigt sich u.a. darin, wie Studierende auf die betreffenden Konzepte referieren, z.B. in ihren Seminararbeiten. Wird die Universität als ein Raum verstanden, wo Wissen offeriert, aber auch permanent in Frage gestellt wird und Studierende sich Wissen aneignen, ,indem sie dieses vor dem Hintergrund ihrer Deutungsressourcen verstehen und nicht verstehen, auslegen und umformen" (Mecheril/Klingler 2010: 87), so können studentische Arbeiten u.U. seismographisch theoretische Schwierigkeiten aufzeigen, indem sie z.B. mit Missverstehen, Verkürzungen oder Auslassungen darauf reagieren.

In diesem Zusammenhang kann eine Seminararbeit als ein Dokument verstanden werden, in dem sichtbar wird bzw. werden kann, wie Studierende mit Wissensressourcen umgehen, auf unterschiedliche Diskurspositionen verweisen, sie verarbeiten und in ihre eigene Arbeit einbringen - oder aber eben auch nicht verarbeiten und nicht einbringen. Daran zeigt sich nicht nur aus einer Entwicklungsperspektive die schrittweise Sozialisierung im Fach, sondern auch auf einer theoretischen Ebene, wo Konzepte im Fachdiskurs offenbar schwer fassbar sind.

In den Arbeiten Studierender lassen sich Missverständnisse aufzeigen und Vorannahmen verorten, die von vielen als so selbstverständlich genommen werden, dass sie manchmal auch dann nicht in Frage gestellt werden, wenn Seminarinhalte oder Fachtexte diesen Positionen zuwiderlaufen und dadurch eine Reaktion hätten hervorrufen müssen: Eine solche Reaktion kann entweder eine Revision der ursprünglichen Position sein oder der Versuch, (neue) Argumente zu finden, um sie zu verteidigen. Es zeigt sich aber zuweilen eine dritte Form der Reaktion: Inhalte, die der ursprünglichen Position zuwiderlaufen, werden schlicht beiseitegelassen, möglicherweise als verkomplizierendes Detailwissen verstanden, auf das man in der Arbeit nicht unbedingt eingehen muss.

In diesem Kontext ist der vorliegende Beitrag zu sehen: Es soll analysiert werden, wie „Kultur" in einer BA-Arbeit vertextet wird, wie sich der „herkömmliche“ Kulturbegriff hartnäckig gegenüber dem Input hält, der ihm zuwiderläuft (Seminarlektüre, Feedback) - und welche Konsequenzen diese Komplexitätsreduktion für die ganze Arbeit hat. Dabei geht es nicht darum, zu zeigen, was an der Arbeit missglückt ist, sondern vielmehr um das, was dahintersteckt: Eine Persistenz von homogenisierenden Vorannahmen über „Kultur“, die dazu geführt haben, dass Erkenntnispotential verschenkt worden ist. Es geht also zentral um einen Knackpunkt des Diskurses über „Kultur“ - nämlich die unzulässige Verallgemeinerung, die hinter der Ethnisierung von „Kultur“ steckt. Dabei lohnt es sich, die Frage auch aus der Perspektive studentischer Schreibentwicklung zu beleuchten. Darauf wird im nächsten Abschnitt noch genauer eingegangen.

\subsection{Anforderungen einer BA-Arbeit vor dem Hintergrund der Schreibentwicklung im wissenschaftlichen Schreiben}

Schreiben im Studium bedeutet einerseits Schreiben um zu lernen und zu verstehen - aber andererseits auch um zu beweisen, dass gelernt und verstanden wurde. Seminar-, Bachelor- oder Masterarbeiten sind typische Beispiele für eine solche Doppelfunktion. Einerseits dient das Schreiben dem Lernen und Üben: Die Studierenden erarbeiten selbstständig ein Thema, arbeiten sich 
ein, nützen die epistemische Funktion des Schreibens (vgl. Molitor 1985: 335), also das Schreiben als Instrument des Denkens, und erarbeiten sich dadurch Inhalte. Dabei sammeln sie Erfahrung im wissenschaftlichen Schreiben sowohl auf der Prozessebene (im Hinblick auf Strategien, Routinen und Schreibprozessorganisation) als auch auf der Produktebene (im Umgang mit entsprechenden Textsortenkonventionen der Diskursgemeinschaft). Andererseits dient das Schreiben aber auch dem Nachweis von Wissen und Kompetenzen: Hausarbeiten sind eben auch Prüfungsformate. In Bachelor- oder Masterarbeiten geht es darüber hinaus darum, einen ,auch inhaltlich eigenen Beitrag innerhalb der Disziplin zu leisten“ (Schindler 2017: 110). Dafür müssen sich Studierende einen Überblick über Diskurspositionen in ihrer Fachdisziplin verschaffen; das wissenschaftliche Schreiben erfordert „discipline-specific knowledge of how professionals conceptualize issues and talk about them in order to achieve their disciplinary and professional goals“ (Bathia 2015: 9).

Einen solchen Überblick über diese Konzeptualisierungen - in ihrem jeweils spezifischen fachlichen und historischen Kontext - zu gewinnen ist eine Sache lebenslangen Lernens. Drei Jahre BA-Studium sind im Vergleich dazu verhältnismäßig kurz. In der BA-Arbeit eine eigene, stringente Argumentation zu vertreten, die souverän Position bezieht im „diskursiven Gewimmel“ (Jäger/Jäger 2007: 25) des Fachs, stellt also hohe Anforderungen an die Studierenden, sowohl im Hinblick auf die quantitative Anhäufung von Wissen, als auch im Hinblick auf die qualitativ-heuristische Verarbeitung und die rhetorische Darstellung dieses Wissens.

Je nachdem, wie erfolgreich Studierende neues Wissen implementieren, unterscheiden Yancey et al. (2014: 104) zwischen Assemblage und Remix': Mit Assemblage ist dabei ,an unsuccessful use of prior knowledge" gemeint, bei dem der Misserfolg darin besteht, dass Studierende auf bereits Bekanntes zurückgreifen und es mit einer limitierten Menge von neuem Wissen kombinieren, wodurch eine teilweise inkonsistente Wissensbasis entsteht. Beim Remix hingegen wird das Wissen stärker verarbeitet und neu geordnet: „by reworking and integrating prior knowledge and practice with new knowledge and practice as they assess new tasks" schaffen die Studierenden „a more successful use of prior knowledge“ (2014: 104). Remix ist also ähnlich zu verstehen wie Abduktion, als "gelingende[r] Versuch, Ordnung in das Chaos zu bringen" (Ortner 2000: 31). Ein weiterer, darüber hinausgehender Schritt wäre nach Yancey et al. (2014: 104) der Aufbau von neuen Wissensstrukturen („,reating new knowledge and practice“). Dies geschieht häufig durch ,a setback or critical incicent" - nämlich, wenn das vorhandene Wissen merkbar nicht ausreicht, um eine Aufgabe zu erfüllen und es deshalb zu einem (vorübergehenden) Scheitern kommt, das sich aber schließlich als ein produktives Scheitern erweisen kann, wenn es dazu führt, dass neue Wissensstrukturen aufgebaut und neue Praktiken erlernt werden (vgl. Yancey et al. 2014: 104). Inwieweit das Schreiben über „Kultur' Ansatzpunkte für solche „setbacks“" oder „,critical incidents" bietet, wird zu diskutieren sein.

Die Unterscheidung von Assemblage und Remix/Creating new knowledge erinnert ansatzweise an die Unterscheidung Knowledge Telling und Knowledge Transforming (vgl. Bereiter/Scardamalia 1987). Für die Verarbeitung und Darstellung von Kulturkonzepten in wissenschaftlichen Texten ist zumindest „Knowledge Transforming“ (Bereiter/Scardamalia 1987: 10ff) notwendig, also nicht nur die Wiedergabe von Wissen („Knowledge Telling“, Bereiter/Scardamalia 1987: 10), sondern eine selbstständige und tiefergehende Auseinandersetzung mit diesem Wissen, das idealerweise mittels „Knowledge Crafting“ (Kellogg 2008: 4) dann auch noch möglichst professionell und den Konventionen der Diskursgemeinschaft entsprechend dargestellt werden soll. Bereiter/ Scardamalia (1987) und Kellogg (2008) beziehen die Konzepte Knowledge Telling/Transforming/ Crafting ausschließlich auf das Schreiben, bei Bereiter/Scardamalia (1987) geht es dabei mehr um die Komplexität des Schreibprozesses, während Kellogg (2008) vor allem eine Entwicklungsperspektive einnimmt.

1 Yancey et al. beziehen sich dabei in erster Line auf Vorannahmen über das Schreiben und Schreibprozesse. Das Konzept lässt sich aber auch auf andere Gegenstände übertragen. 
Wenn Studierende über eine eigene Fragestellung im Bereich der Transkulturellen Kommunikation schreiben, wird von ihnen erwartet, dass sie dies vor dem Hintergrund des aktuellen Fachdiskurses tun. Dabei haben sie sowohl heuristische als auch rhetorische Anforderungen (vgl. Dengscherz 2018) zu bewältigen: Auf der heuristischen Ebene müssen sie Muster erkennen und Zusammenhänge im Diskurs verstehen und daraus Schlüsse für ihre eigene Arbeit ziehen. Auf der rhetorischen Ebene müssen sie adäquate Begriffe und Formulierungen finden, um tatsächlich das auszudrücken, was sie sagen möchten. Wie Studierende mit diesen Anforderungen umgehen und wie sie sie bewältigen, lässt sich einerseits ausgehend von Erfordernissen bestimmter Schreibaufgaben betrachten (vgl. Beaufort/Iñesta 2014), andererseits aus einer Entwicklungsperspektive wie dies Steinhoff (2007) und Pohl (2007) tun. Steinhoff (2007) fokussiert vor allem auf wissenschaftliche Formulierungskompetenz, also auf die rhetorische Ebene der ,alltäglichen Wissenschaftssprache“ (Ehlich 1999). Steinhoff beschreibt dabei vier Entwicklungsstufen, die von Studierenden durchlaufen werden. Auf der ersten Stufe, der Transposition, versuchen Lernende „neue Probleme mit alten Mitteln zu lösen“ (Steinhoff 2007: 139), indem sie auf bekannte Formulierungen aus anderen Domänen (z.B. dem schulischen Aufsatzunterricht oder dem Journalismus) zurückgreifen. Auf der zweiten Stufe, der Imitation, haben Studierende bereits Formulierungsmuster im wissenschaftlichen Schreiben erkannt und versuchen nun, sie nachzuahmen. Dies führt häufig zu übertriebenem Nominalstil, unübersichtlichen Satzgefügen und einem ,überzogenen“, „gespreizten“ Sprachgebrauch, die Stilmittel wissenschaftlichen Schreibens werden ,noch nicht hinreichend kontrolliert" (Steinhoff 2007: 143f). Auf der Stufe der Transformation haben die Studierenden begonnen, sich wissenschaftssprachliche Mittel anzueignen und sie sukzessive selbstständig anzuwenden. Dabei kommt es aber noch zu „Ausdrucks- und Formulierungsbrüchen“ (Steinhoff 2007: 147), die charakteristisch sind für dieses Übergangsstadium, bis die Lernenden die vierte Stufe, nämlich die Kontextuelle Passung erreichen: Ihre „Textäußerungen schließen“ nun ,an den Usus der Wissenschaftskommunikation an“ (Steinhoff 2007: 148). Auf den präkonventionellen Sprachgebrauch folgt also der konventionelle - und sehr viel später in der Schreibentwicklung dann auch noch ein postkonventioneller, wenn erfahrene Wissenschaftler*innen beginnen, allmählich mit Konventionen zu spielen und sich zuweilen auch über sie hinwegsetzen (vgl. Steinhoff 2007: 139). Die Akzeptanz von wissenschaftlichem Sprachgebrauch hat also zu einem gewissen Grad also auch mit der (Macht-)Position in der Diskursgemeinschaft zu tun (vgl. Knappik 2017: 58).

Während sich Steinhoff (2007) vor allem auf Textsortenkonventionen auf der rhetorischen Ebene sprachlicher Gestaltung bezieht, schließt Pohl (2007) stärker makrostrukturelle Aspekte mit ein und setzt sich explizit mit dem Problem der „Strittigkeit“ (374) unterschiedlicher Diskurspositionen in der Fachliteratur auseinander. Die Entwicklungsniveaus, die Pohl beschreibt, verortet er auch im Hinblick auf den Umgang mit solcher Strittigkeit. Dieser Aspekt ist für die vorliegende Analyse wesentlich.

Pohl (2007: 396) nimmt drei Entfaltungsniveaus wissenschaftlichen Argumentierens an. „Argumentativer Dissens" wird auf dem ersten Entfaltungsniveau erst gar nicht eröffnet, auf dem zweiten Entfaltungsniveau zwar eröffnet, allerdings ohne Folgen für den Gesamttextaufbau und auf dem dritten Entfaltungsniveau auf eine Weise eröffnet, dass sich dies auch auf den Gesamttextaufbau auswirkt. Auf der ersten Stufe zieht also „Strittigkeit (egal ob fingiert oder real) keine makrostrukturellen Effekte nach sich“(Pohl 2007: 374). Unterschiedliche Positionen bzw. „Stimmen aus der Literatur stehen zueinander in einem unisonen Gleichklang und ergänzen sich allenfalls aspektuell“ (374f). Auf der zweiten Stufe wird ,argumentative[r] Dissens“ bereits thematisiert, der „Zugriff auf die verschiedenen Positionen“ erfolgt aber , in erster Linie isoliert auf die betreffenden Konzepte, Ansätze, Theorien als solche, ohne deren argumentative Stützungen einzubeziehen“ (375). Erst auf der dritten Stufe werden „Dissenskonstellationen [...] nicht nur lokal argumentativ ausgefochten, sondern haben makrostrukturelle Organisationseffekte“. Und erst auf dieser Stufe wird auch die Anordnung von Informationen nach einer argumentativen bzw. konklusiven Struktur vorgenommen. 
Schindler (2017) macht darauf aufmerksam, dass Pohl (2007) im Gegensatz zu Steinhoff (2007) nicht davon ausgeht, dass die Entwicklungsniveaus überwunden werden: „Sie gehen vielmehr ineinander auf bzw. führen zu einem erweiterten Verständnis der eigenen Schreibanforderungen, aber auch der Gestaltungsmöglichkeiten“" (Schindler 2017: 111). Dies zeigt sich auch in der Analyse der vorliegenden Arbeit, wo sich unterschiedliche Entwicklungsniveaus gleichzeitig zeigen, die jeweils an unterschiedlichen Textstellen festgemacht werden können.

Die von Steinhoff (2007) und Pohl (2007) skizzierten Entwicklungsniveaus stellen einen produktiven und erhellenden Ausgangspunkt für die vorliegende Analyse dar (vgl. auch Abschnitt 4 zur Diskussion der Ergebnisse). Der Fokus meines Beitrags liegt allerdings weniger auf der makrostrukturellen Textorganisation (wie bei Pohl) oder der mikrostrukturellen Erfüllung von Textsortenkonventionen (wie bei Steinhoff), sondern es geht mir darum zu zeigen, wie auf der inhaltlich-heuristischen Ebene mit unterschiedlichen Kulturbegriffen operiert wird.

\section{Datenbasis, Analysefokus und methodisches Vorgehen}

In einer exemplarischen Einzelfall-Analyse soll nun der Frage nachgegangen werden, wie ein Schreiber im BA-Studium Transkulturelle Kommunikation am Zentrum für Translationswissenschaft (ZTW) der Universität Wien mit den heuristischen und rhetorischen Anforderungen (und Herausforderungen) umgeht, die sich für ihn beim Schreiben über „Kultur“ ergeben. Die Arbeit beschäftigt sich mit Stereotypen und Vorurteilen und erscheint dadurch für eine Analyse des Umgangs mit dem Kulturbegriff besonders geeignet: Zum einen sind Stereotype und Vorurteile auf Generalisierung, Essentialisierung und Homogenisierung zurückzuführen und haben damit einiges mit dem unreflektierten Umgang mit ,Kultur' zu tun (auf dem sie auch häufig beruhen). Zum anderen bieten die eingangs diskutierten, differenzierten Kulturkonzepte nützliche Anhaltspunkte, um die Entstehung von Vorurteilen zu verstehen. Es ist damit eine zentrale und wichtige Frage, inwieweit es dem Studenten gelingt, jene Konzepte, die während des Semesters im Seminar diskutiert worden sind, auch in seiner Arbeit zu berücksichtigen - und welche Folgen es für den Erkenntnisprozess hat, wenn er das nicht (oder nur teilweise) tut. Im Zentrum der Analyse steht die erste Version der BA-Arbeit (V1), als Kontext werden die Endversion (EV), zwei Konzeptversionen im Vorfeld (K1 und K2) sowie zusätzliche Informationen einbezogen, die der Schreiber per E-Mail zur Verfügung gestellt hat. Angelehnt an die Qualitative Inhaltsanalyse (vgl. Mayring 2010) und die Kritische Diskursanalyse (vgl. Jäger 2009) wird der Text der BA-Arbeit einer eingehenden Analyse unterzogen. Der Einzelfall wird dabei im ,real-life context" (Yin 2009: 18) betrachtet, also vor dem Hintergrund seines sozialen Kontexts (vgl. Meyer 2016: 78). Der soziale Kontext ist in diesem Fall das Seminar Transkulturelle Kommunikation in seiner Einbettung in das BA-Studium am Zentrum für Translationswissenschaft. Daraus lassen sich entsprechende Kontextinformationen gewinnen, u.a. über den Input, den der Student (über entsprechende Lektüreempfehlungen) im Hinblick auf die Auseinandersetzung mit dem Kulturbegriff erhalten hat.

Der Schreiber der Arbeit, László2 (23), ist Student am Zentrum für Translationswissenschaft der Universität Wien. Seine L1 ist Ungarisch, seine Bildungssprachen sind Ungarisch und Deutsch. László hat also bereits in seiner Schulzeit in einem Gymnasium in Ungarn bildungssprachliche Kompetenzen auf Deutsch erworben, 2014 die Matura abgelegt und dann das BA-Studium Transkulturelle Kommunikation mit den Arbeitssprachen Ungarisch, Deutsch und Englisch absolviert. László ist ein erfolgreicher Student, schreibt bereits im 6. Semester seine BA-Arbeit und schließt das BA-Studium in Mindeststudienzeit ab. In seiner BA-Arbeit beschäftigt er sich mit einem Thema aus dem Feld der Transkulturellen Business-Kommunikation, der Titel seiner Arbeit lautet: „Stereotype und Vorurteile an Bord. Selbstbild und Fremdbild der ungarischen FlugbegleiterInnen bei Qatar Airways“.

2 Name geändert. 
Die Arbeit ist im Wintersemester 2017/18 in meinem Seminar „Transkulturelle Kommunikation" am ZTW entstanden. In ihren BA-Arbeiten sollten die Studierenden eine selbst gewählte Fragestellung (empirisch) untersuchen. Unterstützung erhielten sie über Feedback zu verschiedenen Stadien der Idee und der Arbeit sowie durch Input aus Fachtexten aus dem Bereich der Transkulturellen Kommunikation. Im Seminar wurden Texte und Textausschnitte gelesen, die sich dem Thema „Kultur“ aus unterschiedlichen Perspektiven nähern, über den Kulturbegriff und dahinterliegende Konzepte wurde wiederholt diskutiert, zunächst aus theoretischer Perspektive, dann anhand der konkreten Fragestellungen, die die Studierenden in ihren Arbeiten behandeln wollten. Im ersten Drittel des Semesters war ein Konzept für die geplante Arbeit auszuarbeiten und abzugeben, in der zweiten Hälfte des Semesters sollten die Studierenden den Stand ihrer Arbeit (Work in Progress) in einem Kurzvortrag oder einem Poster präsentieren und sich darüber mit ihren Studienkolleg*innen über ihr Thema und ihre Herangehensweise austauschen. Dazu gehörte auch die Reflexion über verschiedene Methoden der wissenschaftlichen Datenerhebung und Datenanalyse (vor allem Interviews und Fragebögen). Im Zuge dessen wurden bei einigen Präsentationen auch Fragebögen-Entwürfe an die Gruppe verteilt und Frageformate diskutiert. Dabei ging es nicht selten um die Validität einzelner Fragen und um eventuelle Vorannahmen, die in einer Frage zum Ausdruck kommen. Zusätzliches Feedback gab es - auf Wunsch und bei Bedarf - in den Sprechstunden.

Die Diskussion der Fragebögen in der Gruppe war für die meisten Studierenden, die eine Fragebogenstudie durchführten, ein wesentlicher Bestandteil der Fragebogenentwicklung. László hatte allerdings ursprünglich geplant, die Daten für seine Arbeit mit qualitativen Interviews zu erheben und sich erst später für eine Fragebogenstudie entschieden. Dies hat mit dazu geführt, dass er seinen Fragebogen nicht im Seminar präsentiert hat und dieser auch nicht in der Gruppe diskutiert worden ist. Andere Formen des Feedbacks hat László in Anspruch genommen: Feedback zum Konzept in der Sprechstunde und ein „Zwischenfeedback“ zu einer 1. Version der fertigen Arbeit ${ }^{3}$.

Für die Analyse wird ebendiese 1. Version herangezogen, die László für das „Zwischenfeedback" eingereicht hat. Diese Version kann als besonders ,,authentisch“ (Mayring 2010: 30) gelten, authentisch in dem Sinne, dass sie noch weniger von meinem Feedback beeinflusst ist als die Endversion, allerdings doch - zumindest ansatzweise, wie zu sehen sein wird - vom Input im Seminar selbst (Lektüre von Fachtexten, Problematisierung von homogenisierenden/ethnisierenden Zugängen, s.o.). Diese Einflussfaktoren werden in der Analyse mitreflektiert und es wird dabei vor allem auf die Frage fokussiert, wie László mit diesen (neuen) Einflüssen umgeht, und wie sie mit eventuellen anderen Einflüssen aus Fachliteratur, Studium oder Alltag in Konflikt treten.

Als Seminarleiterin und Wissenschaftlerin stehe ich dieser BA-Arbeit in einer Doppelrolle gegenüber. Zum Zeitpunkt der Analyse ist die Arbeit allerdings bereits benotet, und der Student befindet sich in keinerlei Abhängigkeitsverhältnis, sodass sich hier kein forschungsethisches Problem ergibt. Für die inhaltliche Analyse ist die erwähnte Doppelrolle teilweise von Vorteil, weil ich die Genese der Arbeit ebenso kenne wie den Ablauf und den Kontext des Seminars. Andererseits kann sich genau dieser Umstand aber auch wieder nachteilig auswirken, weil die eigene Erinnerung an Ereignisse oder Diskussionen des laufenden Semesters die Analyseergebnisse beeinflussen könnte. Um dem entgegenzuwirken, soll die Analyse vor allem vor dem Hintergrund der Pflichtlektüre aus dem Seminar vorgenommen werden. Während des Semesters wurden drei Texte zum Kulturbegriff im Plenum kritisch diskutiert, nämlich: Hormel/Jording (2016), Layes (2005) und Saal (2014). Die beiden letzteren zitiert László auch in seiner BA-Arbeit, auf Hormel/ Jording (2016) verweist er aber nicht.

3 Wenn die Studierenden ihre Arbeit bis zu einem bestimmten Termin abgeben, können sie auf Wunsch ,Zwischenfeedback" erhalten. Das bedeutet, dass die Arbeit nicht gleich benotet wird, sondern noch einmal überarbeitet werden kann und in der Endversion eventuelle Monita behoben werden können. Im WS 2017/18 haben 8 von 34 Studierenden diese Möglichkeit in Anspruch genommen, László war einer von ihnen. 
Im Folgenden soll nun die Thematik und Genese der Arbeit im Seminarkontext verortet und auch die Arbeit selbst in drei Schritten analysiert werden: Für einen ersten Überblick wird quantitativ festgestellt, in welcher Häufigkeit Begriffe aus dem Umfeld „Kultur“ verwendet werden (Abschnitt 3.1). Die Häufigkeitsanalyse bietet einen nützlichen Ausgangspunkt für die qualitativhermeneutische Vertiefung im Hinblick auf den Umgang mit dem Kulturbegriff und die darunterliegenden Konzepte von „Kultur“. Die anschließende qualitative Analyse folgt den folgenden Leitfragen:

- Wie und in welchen Kontexten wird in der Arbeit über „Kultur“ geschrieben?

- Welche lexikalischen Mittel werden dafür verwendet und wie sind sie eingebettet?

- Inwieweit lassen sich Rückschlüsse auf Konzepte und Diskurspositionen ziehen, die den jeweiligen Textstellen zugrunde liegen?

Vor dem Hintergrund dieser Fragen werden nun der theoretische Teil der Arbeit (Abschnitt 3.2) und die empirische Untersuchung, insbesondere die Konzeption des Fragebogens (Abschnitt 3.3.) eingehender analysiert.

\section{Exemplarische Analyse der BA-Arbeit}

In Lászlós BA-Arbeit geht es um internationale Business-Kommunikation im Flugverkehr. Als Grund für die Themenwahl „Stereotype und Vorurteile an Bord. Selbstbild und Fremdbild der ungarischen FlugbegleiterInnen bei Qatar Airways" nennt László familiäre Kontakte, die ihm ermöglicht haben, immer wieder mit Qatar Airways zu reisen und dabei „Verhaltensweisen, Reaktionen, Interaktionen und prompte Konfliktlösungsfähigkeit der FlugbegleiterInnen zu beobachten“ (E-Mail vom 24.3.2018). Es ging László also darum, Phänomene aus der internationalen Business-Kommunikation, die er selbst auf seinen Reisen mit Qatar Airways beobachtet hat, nun systematisch wissenschaftlich zu untersuchen.

Im Mittelpunkt steht dabei die Interaktion vor dem Hintergrund potentieller Vorurteile und Stereotype und die Frage, wie letztere durch ebendiese Interaktion abgebaut werden können. Lászlós Perspektive auf das Thema erinnert stark an Interkulturelle Trainings, so wie Castro Varela (2010) sie - kritisch - umreißt:

Interkulturelle Trainings stehen wieder - oder immer noch - hoch im Kurs. Das kann kaum verwun-
dern, denn ihre Versprechen sind sehr attraktiv: gute Kommunikation zwischen Menschen aus unter-
schiedlichen „Kulturen“, effiziente Arbeit in transnationalen Teams etc. Interkulturelle Trainings fü-
gen sich sanft in einen Diskurs ein, der von Internationalisierung und Europäisierung spricht und Mo-
bilität als das wichtigste Ziel pädagogischen Tuns zu setzen scheint. (Castro Varela 2010: 117)

László geht es ebenfalls um gute Kommunikation, darüber hinaus auch darum, wie durch Interaktion Stereotype und Vorurteile abgebaut werden können. Die Vorannahme, auf der seine Arbeit basiert, ist, dass Menschen in unterschiedliche abgrenzbare „Kulturen“ hineingeboren und dort sozialisiert (=> geprägt) sind und dass es deshalb in der Kommunikation zwangsläufig zu Missverständnissen und Problemen kommt, denen dann wiederum mit professioneller Kommunikation begegnet werden kann und soll.

Die Analyse zeigt, wie diese - durchaus weit verbreitete - auf Othering ${ }^{4}$ beruhende Vorannahme in Lászlós Arbeit zu einer problematischen Komplexitätsreduktion und zu Verzerrungen der empirischen Studie führt. Es wird zu sehen sein, wie sich die Vorannahme als persistent sowohl gegenüber der Lektüre und den Diskussionen im Seminar als auch gegenüber seinen eigenen empirischen Ergebnissen erweist und wie dies den Erkenntnisprozess erschwert. Da es sich um die Analyse eines - in der vorliegenden BA-Arbeit nicht gelösten - Problems handelt, ist eine gewisse Defizitorientierung nicht ganz zu vermeiden. Wichtiger ist aber dabei die Entwicklungsper-

4 Zur Begriffsgeschichte und -vorgeschichte vgl. Thomas-Olalde/Velho (2011). 
spektive: Es soll reflektiert werden, welche Umstände dazu beigetragen haben könnten, dass das Problem nicht gelöst worden ist, und welche Anforderungen bzw. Herausforderungen László hätte bewältigen müssen, um es zu lösen. Darüber hinaus soll exemplarisch gezeigt werden, wie das - immer noch weit verbreitete - homogenisierend-ethnisierende Kulturkonzept in einer konkreten Fragestellung sowohl die Konzeption der empirischen Untersuchung als auch ihre Auswertung so stark beeinflussen kann, dass dies die Validität der Untersuchung gravierend beeinträchtigt.

\subsection{Zum Begriffsinventar der BA-Arbeit}

Die Analyse der Häufigkeit einzelner Lexeme in der Arbeit sagt bereits einiges über die thematisch-lexikalische Schwerpunktsetzung aus. Die 1. Version der BA-Arbeit (V1) umfasst insgesamt 41 Seiten bzw. 11.619 Wörter (inkl. Deckblatt, Selbstständigkeitserklärung, Inhaltsverzeichnis, Bibliographie und Fragebogen im Anhang).

Für die folgende Häufigkeitsanalyse wurde ausschließlich der Fließtext (inkl. Kapitelüberschriften) herangezogen. Deckblatt, Selbstständigkeitserklärung, Inhaltsverzeichnis, Bibliographie und Fragebogen wurden ausgeklammert. Eine Einbeziehung von Deckblatt und Inhaltsverzeichnis hätte zu wenig aussagekräftigen Doppelungen geführt, die Selbstständigkeitserklärung wurde als Baustein von außen übernommen, und die Bibliographie besteht aus Titeln anderer Autor*innen. Es scheint also sinnvoll, diese Teile nicht mit in die Häufigkeitsanalyse aufzunehmen. Der Fragebogen liegt wiederum in einer anderen Sprache vor als der Fließtext, nämlich auf Ungarisch. Der Umgang mit dem Kulturbegriff im Fragebogen wird in einem eigenen Abschnitt reflektiert.

Der Fließtext (inkl. Kapitelüberschriften) der V1 umfasst 28 Seiten und besteht aus 11.619 Wörtern. Die thematisch-lexikalische Schwerpunktsetzung lässt sich gut in einer Wortwolke visualisieren:

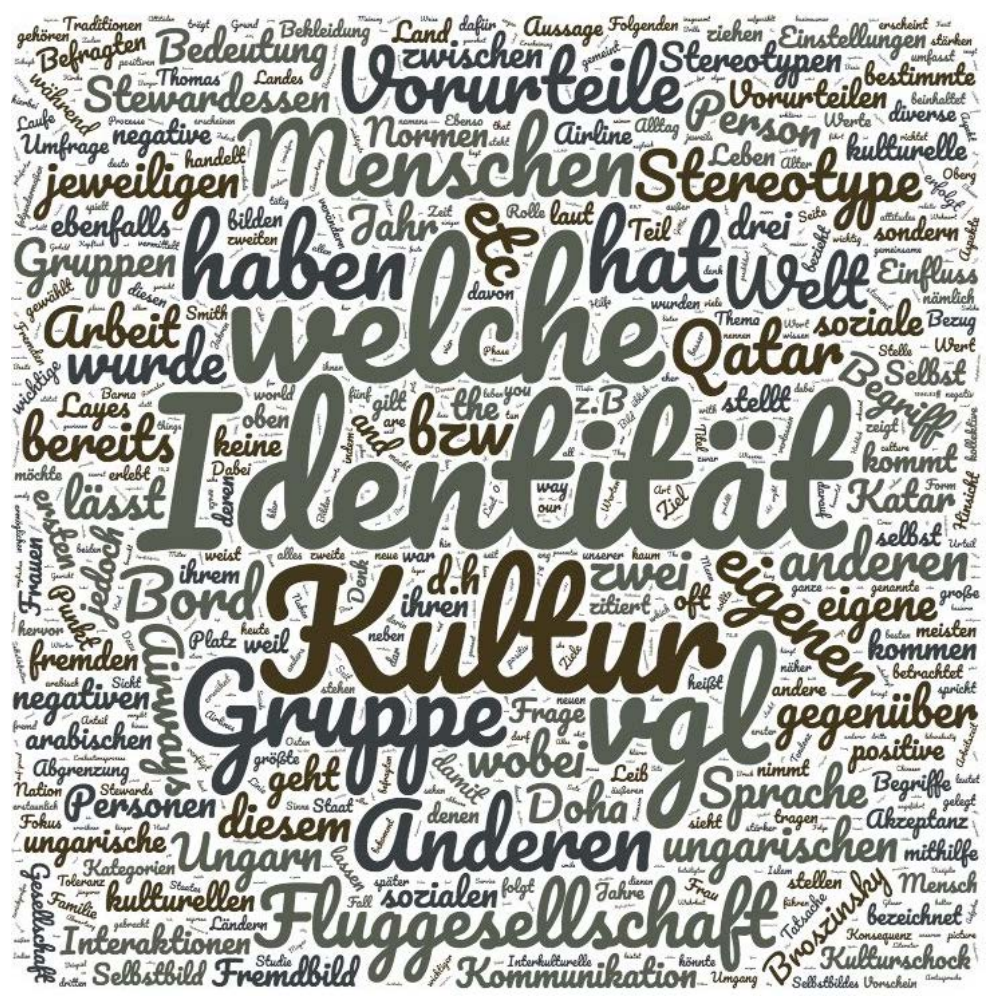

Abb. 1: Wortwolke aus dem Fließtext der BA-Arbeit (V1)

5 Die Wortwolke wurde mit Hilfe des kostenlosen Online-Tools https://www.wortwolken.com/ erstellt. [30.03.2018]. 
„Identität“ und „Kultur“ erscheinen hier als die ganz offensichtlich häufigsten Begriffe. Dies bestätigt auch die Ermittlung der Worthäufigkeit in MaxQDA: „Identität“ (58 Nennungen) und „Kultur“ (46 Nennungen) sind die häufigsten bedeutungstragenden Lexeme in der Arbeit. „Identität“ belegt dabei Platz 19 bei der Gesamtzählung, „Kultur“ Platz 29. Die Plätze davor und dazwischen werden ausschließlich von Wörtern mit vorwiegend grammatischer Bedeutung belegt (Platz 1 bis 18: die, der, und, sich, in, den, auf, mit, ist, eine, von, das, werden, zu, als, man, dass, Die; Platz 20 bis 28: sie, sind, einer, dem, kann, des, wie, welche, wird).

Die besonders häufigen Wörter mit grammatischer Bedeutung sind tendenziell kurz: Sie zählen zwischen 3 und 6 Buchstaben. Werden also alle Wörter mit weniger als 6 Buchstaben von der Zählung ausgeschlossen, fällt ein Großteil dieser (im Hinblick auf den thematischen Schwerpunkt der Arbeit wenig aussagekräftigen) Wörter weg, und es lässt sich deutlicher auf semantische Aspekte fokussieren. Bei einer neuerlichen Häufigkeitszählung landet nun „Identität“ auf Platz 2 und „Kultur“ auf Platz 4. Davor und dazwischen stehen nur noch zwei Wörter mit vorwiegend grammatischer Bedeutung, nämlich „werden“ und „welche“. Die häufigsten Lexeme mit mindestens 6 Buchstaben sind im Fließtext der Arbeit die folgenden (Häufigkeit in Klammern): werden (82), Identität (58), welche (49), Kultur (46), können (39), Gruppe (32), Menschen (30), Anderen (26), Vorurteile (24), eigenen (23), Fluggesellschaft (23), Stereotype (22), FlugbegleiterInnen (20), anderen (19), ungarischen (18), bereits (17), eigene (16), gegenüber (16), Airways (15), Arbeit (15).

Bei der Aufzählung zeigt sich bereits, dass bei der automatisierten Auswertung der Worthäufigkeit in MaxQDA auch unterschiedliche Wortformen und Schreibweisen berücksichtigt - und getrennt gezählt werden (Andere/andere; die/Die). Dies kann das Ergebnis etwas verzerren. Was ebenfalls weder in der Wortwolke, noch in der automatischen Gesamtzählung der Häufigkeiten zu sehen ist, ist die Produktivität einzelner Begriffe, die auch in unterschiedlichen Zusammensetzungen vorkommen können. Dies gilt z.B. für den Begriff „Kultur““

Während „Kultur“ als Einzelbegriff im Singular ,nur“ 46 Mal im Fließtext der Arbeit vorkommt, erweist sich der Begriff in seiner Gesamtproduktivität noch wesentlich präsenter in der Arbeit. Werden auch alle Wortverbindungen mit Kultur/-kultur/kultur- mitgezählt, können bereits 136 Nennungen im Fließtext gezählt werden. Interessant ist, dass dabei „Transkulturalität" oder „transkulturell“ überhaupt nicht vorkommen. ${ }^{6}$ Die folgende Tabelle veranschaulicht, in welchen Wortverbindungen -kultur- in Lászlós Arbeit (V1) enthalten ist:

\begin{tabular}{|c|l|}
\hline Häufigkeit & Begriff \\
\hline 53 & Kultur/Kulturen ${ }^{7}$ \\
\hline 25 & kulturell \\
\hline 25 & interkulturell \\
\hline 11 & Kulturschock \\
\hline 5 & multikulturell \\
\hline 4 & Kulturbegriff \\
\hline 2 & Enkulturation \\
\hline 2 & kulturális $^{8}$ \\
\hline 2 & Kulturvielfalt \\
\hline 1 & Fremdkultur \\
\hline 1 & fremdkulturell \\
\hline
\end{tabular}

6 Das Adjektiv „transkulturell“ kommt nur auf dem Deckblatt vor, in der Bezeichnung der Lehrveranstaltung, in deren Rahmen László seine Arbeit geschrieben hat (dem Seminar „Transkulturelle Kommunikation“).

7 46x „Kultur“ im Sg., 7x „Kultur“ im Pl.

8 Ung. für „kulturell“; kommt in einem Zitat vor. 


\begin{tabular}{|c|l|}
\hline 1 & Hochkultur \\
\hline 1 & Kulturgrenzen \\
\hline 1 & Kulturkreis \\
\hline 1 & Kulturwissen \\
\hline 1 & Kulturzugehörigkeit \\
\hline 136 & \\
\hline
\end{tabular}

Tab. 1. Produktivität des Begriffs „Kultur“ im Fließtext der Arbeit

In den folgenden beiden Abschnitten wird nun genauer darauf eingegangen, wie László mit diesem Begriffsinventar umgeht und auf welche Konzepte er sich dabei jeweils - explizit oder implizit - bezieht.

\subsection{Zur theoretischen Reflexion von Kulturkonzepten in der Arbeit}

Lászlós Arbeit basiert auf einem extensional weit gefassten (und teilweise auch nicht ganz geklärten) Kulturkonzept, das Othering reproduziert, und das offensichtlich weder durch diesem Konzept zuwiderlaufende Fachtexte bzw. Diskussionen im Seminar noch durch Feedback ins Wanken gebracht wurde. Hinsichtlich anderer Aspekte, wie Aufbau, Darstellung von Methode und Ergebnissen o. Ä. hat László Anregungen aufgegriffen und umgesetzt, auch selbstständig weiter vertieft. Nur in Bezug auf den Kulturbegriff blieben Interventionsversuche weitgehend wirkungslos bzw. haben nur zu oberflächlichen Korrekturen, aber zu keiner weiterführenden Reflexion geführt.

So war bereits in Lászlós Konzept zu ahnen, dass es Probleme mit Vereinfachungen und Verkürzungen im Hinblick auf den Kulturbegriff geben könnte, so war u.a. die Rede von ,fremdkulturellen Menschen“" (ohne Anführungszeichen) (K1: 2). Auf eine diesbezüglich kritische Anmerkung hin wurden daraus in der zweiten Konzeptfassung „Andere“ (mit Anführungszeichen). Solche Anführungszeichen finden sich dann auch an mehreren Stellen in der Arbeit.

Die Reflexion - oder vielmehr Definition - des Kulturbegriffs spielt in Lászlós BA-Arbeit durchaus eine Rolle. Das Kapitel „Ich und die ,Anderen““ umfasst in der ersten Version (V1) gut 11 Seiten (von 28 Seiten Fließtext), davon sind aber nur eineinhalb Seiten der Auseinandersetzung mit dem Kulturbegriff gewidmet. Die folgende Übersicht zeigt die Einteilung der BA-Arbeit in Kapitel und Unterkapitel (V1:3): 


\section{Inhaltsverzeichnis}

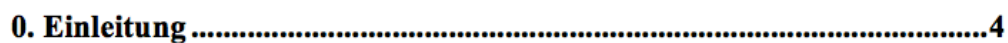

1. Ich und die „Anderen“ ................................................................ 5

1.1 Was ist Kultur? - eine kleine Einführung........................................

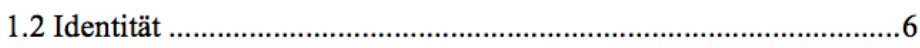

1.2.1 Selbstbild, Fremdbild ................................................... 8

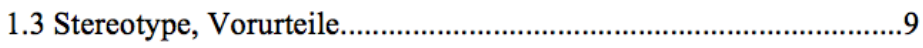

1.3.1 Stereotype - Definition, Entstehung ..................................10

1.3.2 Vorurteile - Definition, Entstehung..................................12

1.3.3 Attitüde........................................................................ 14

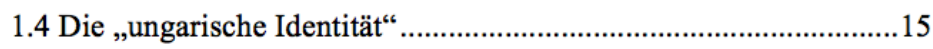

1.4.1 Attitüde gegenüber „Fremden“ .........................................16

2. Als ungarische(r) ElughegleiterIn bei Qatar ........................................17

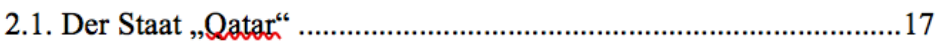

2.2. Qatar Airways .............................................................................. 19

2.2.1 Geschichte .....................................................................19

2.2.2 Voraussetzungen, Erwartungen.....................................20

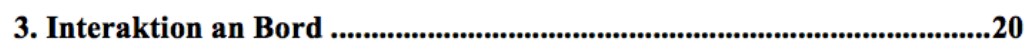

3.1. Kulturvielfalt an Bord - interkulturelle Kommunikation ...............21

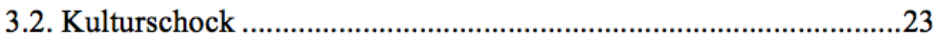

4. Resultat der Interaktion mit ,Anderen"...............................................24

5. Bibliographie.................................................................................................32

\section{Anhang}

Abb. 2. Inhaltsverzeichnis der BA-Arbeit (V1)

Es ist zu sehen, dass László sich, ganz nach guter wissenschaftlicher Praxis, zunächst mit den zentralen Begriffen seiner Arbeit und ihrer Definition in Fachtexten auseinandersetzt. Die Arbeit ist übersichtlich strukturiert, Kapitel und Unterkapitel sind hierarchisiert (stellenweise eröffnet Lázsló aber auch dann eine untergeordnete Ebene, wenn es dann nur ein einziges Unterkapitel gibt). Was in dieser Version der Arbeit noch fehlt, ist ein Methodenkapitel bzw. Methodenreflexion?. Die theoretische Reflexion unterschiedlicher Kulturkonzepte findet vor allem in Kap. 1 und den entsprechenden Unterkapiteln statt.

In Bezug auf den Umgang mit Kulturkonzepten zeigt die Verwendung von Anführungszeichen bei „Anderen“, ,ungarische Identität“ und „Fremden“" schon in den Überschriften der Unterkapitel, dass László die Begriffe - zumindest ansatzweise - problematisiert und ihre Konstruiertheit andeutet. Das hat er aus dem Feedback zum Konzept gelernt.

9 László holt die Reflexion zur Methode Fragebogen in der Endversion (ansatzweise) nach, allerdings auch dort nicht in einem eigenen Unterkapitel, sondern lediglich in einigen Sätzen im Kapitel zu den Resultaten. 
In Kapitel 1 versucht László Fragen rund um Selbstbild und Fremdbild zu klären und bezieht sich auf die „Anderen“ - nicht nur in der Kapitelüberschrift, sondern auch im Fließtext konsequent unter Anführungszeichen. Wenn es um Kultur geht, bezieht er sich allerdings dann doch wieder auf den herkömmlichen Kulturbegriff - was genau genommen nicht zu diesen Problematisierungsansätzen durch die Anführungszeichen passt.

Da László sich mit Stereotypen und Vorurteilen beschäftigen möchte, bietet sich ein kritischer Umgang mit dem Kulturbegriff ganz besonders an. In Kap 1.1. setzt er sich auch explizit mit dem Kulturbegriff und Definitionen davon auseinander. Diese Auseinandersetzung bleibt letztendlich aber an der Oberfläche. Programmatisch dafür ist die folgende Stelle im ersten Absatz des Kapitels:

Wem wir begegnen, kommt in erster Linie darauf an, in welche Kultur wir hineingeboren werden. Schließlich spielt die Kultur die größte Rolle in dem Sozialisierungsprozess. Bevor wir aber tiefer auf die Identität eingehen, gilt es eine Erklärung für den Kulturbegriff zu liefern. (V1: 5)

Kultur ist etwas, wo „wir“ hineingeboren werden und spielt „die größte Rolle in dem Sozialisierungsprozess". Dies erinnert stark an Hofstedes äußerst umstrittenen Kulturbegriff ("collective programming of the mind that distinguishes the members of one group or category of people from others"; Hofstede 2010: 6), der auch im Seminar kritisch diskutiert wurde. László argumentiert später im Kapitel zur Identität auch explizit ,,in Anlehnung an den Kulturbegriff von Hofstede ( $a$ collective phenomenon, 2010: 6)“(V1: 8). Dabei wird dieser Kulturbegriff weder verteidigt noch wird er kritisch betrachtet - er wird nur als gegeben vorausgesetzt. Die Kritik daran wird nicht entkräftet, sie wird in der BA-Arbeit einfach nicht thematisiert.

Noch deutlicher wird die Komplexitätsreduktion im Passus, in dem sich László auf den Text von Saal (2014) bezieht, der eine sehr kritische Auseinandersetzung mit unterschiedlichen Kulturbegriffen und auch eine kritische Auseinandersetzung mit dem Transkulturalitätskonzept enthält. Saal (2014) wird in der BA-Arbeit an einer einzigen Stelle zitiert, und László greift nur die Stelle heraus, in der auf den „herkömmlichen Kulturbegriff“ eingegangen wird:

\footnotetext{
Britta Saal (vgl. 2014²:22) verweist in ihrem Beitrag „Kultur in Bewegung“ auf den herkömmlichen Kulturbegriff: Der Begriff aus dem 18. Jahrhundert hat ursprünglich zwei Bedeutungen. Die erste Bedeutung definiert Kultur als „Hochkultur“, d.h. Kunst, Bildung, Literatur, Theater, Oper etc. Diese Elemente stellen einen wichtigen Teil des Alltagslebens des Bildungsbürgertums sowie der höheren Schichten dar. Die zweite Bedeutung von Kultur bezieht sich auf eine Gruppe von Menschen, die bestimmte Gemeinsamkeiten in ihren Wertvorstellungen und Denkmustern aufweisen wie Geschichte, Sprache und Religion. (V1: 5)
}

Die Problematisierung dieses ,herkömmlichen“ Kulturbegriffs, die bei Saal sogar schon auf derselben Seite 22 beginnt, wird ausgespart. Bei Saal (2014: 22) heißt es an dieser Stelle:

\begin{abstract}
Der zweiten Bedeutung von Kultur, die sich auf Gruppen bezieht, wohnt eine besondere Problematik inne, die dann zum Vorschein kommt, wenn zum Zweck der Abgrenzung eine Homogenisierung des ,Inneren' erfolgt und die Kultur auf diese Weise zu einer statischen, Veränderungen abwehrenden Einheit wird. (Saal 2014: 22)
\end{abstract}

László ,verlässt" Saal allerdings schon vor dieser Problematisierung. Damit nimmt er nicht nur Komplexitätsreduktion vor, sondern verwendet den Text von Saal (2014) in einer Intention, die der Diskursposition des Fachtexts diametral entgegengesetzt ist. Während Britta Saal eine sehr kritische Position gegenüber dem „herkömmlichen“ Kulturbegriff einnimmt, verwendet László ihren Text, um ebendiesen Kulturbegriff zu stützen.

In einem nächsten Schritt wendet László sich einer anderen Definition von Kultur zu, die 1982 auf der „World conference on cultural policies“ in Mondiacult ausgehandelt worden ist und in der sich Anfänge eines Cultural Turn zeigen, indem der Kulturbegriff auf der inhaltlichen Ebene erweitert wird. In Bezug auf die extensionale Dimension bleibt die Definition vage: Verwiesen wird lediglich auf „eine Gesellschaft oder eine soziale Gruppe“(V1: 5). 
Das Problem bei Lászlós Zusammenstellung von Definitionen des Kulturbegriffs liegt nicht zuletzt daran, dass die Fachtexte und Definitionen nicht in ihrer Historizität erfasst werden ${ }^{10}$. Die Problematisierung der extensionalen Dimension von Kultur ist ein verhältnismäßig junges Phänomen, das vor allem durch die Postcolonial Studies (etwa durch die mittlerweile zu „Klassikern“ avancierten Publikationen von Said 1978, Spivak 1988, Bhabha 1994 oder Hall 1994), aber auch die Gender Studies (z.B. Mae/Saal 2014) oder - gerade im deutschsprachigen Raum - auch durch die Migrationspädagogik (z.B. Mecheril/Dirim 2010) getragen wurde und wird und auch in der Translationswissenschaft reflektiert wurde (z.B. Pöchhacker 1994 oder Dizdar 2006). Diese Entwicklungslinien nachzuvollziehen stellt hohe heuristische Anforderungen an die Studierenden. Im Seminar wurden einige ausgewählte theoretische Texte zu Kulturkonzepten eingehend diskutiert (Saal 2014, Hormel/Jording 2016, Layes 2005), eine der Anforderungen an die Studierenden war dabei, diese Texte vor dem Hintergrund ihrer bisherigen (Lektüre-)Erfahrungen im Studium kritisch zu reflektieren und vor diesem Hintergrund einer eigenen empirischen Fragestellung nachzugehen. Dabei war die Reflexion des Kulturbegriffs im Hinblick auf seine Verwendung in den empirischen Studien der Studierenden ein zentrales Thema der Seminardiskussion während des ganzen Semesters.

Dass László versucht, sich die Funktionsweise von Stereotypen und Vorurteilen zu erschreiben (vgl. Knappik 2013: 26) und diese kritisch zu reflektieren, wird mehrfach ersichtlich. So thematisiert er durchaus, dass „Kultur“ dafür herangezogen wird, sich von anderen abzugrenzen und dass das „Orientierungssystem Kultur“ sich auch auf andere Gruppen als Nationen beziehen kann:

Dieses Orientierungssystem, welches für eine Nation, Organisation, Gesellschaft oder Gruppe mit ihren Eigenschaften und Merkmalen (Symbolen, Normen) typisch ist, stärkt das Zugehörigkeitsgefühl des Individuums zu einer Gesellschaft, ermöglicht die Abgrenzung von „Anderen“ und schafft „Handlungsschemata“ für alle Mitglieder der jeweiligen Gesellschaft (vgl. Thomas 2005ª:22). (V1: 6)

Allerdings geht László dann nicht den Schritt weiter, die Konstruktion der Gruppe der „Anderen" zu analysieren und explizit auf die Herausbildung von Stereotypen und Vorurteilen zu beziehen. So kommt etwa der Begriff „Othering" in der Arbeit nicht vor (obwohl er im Seminar eingeführt und diskutiert wurde). Mit der Reduktion auf den herkömmlichen Kulturbegriff verschenkt László das Potential, das - gerade für sein Thema - in neueren Konzepten liegt, die sich mit den Prozessen von Essentialisierung und Homogenisierung auseinandersetzen und dagegen anschreiben. Interessanterweise verwendet László gerade in seinem Kapitel zu Stereotypen und Vorurteilen auch keine Anführungszeichen mehr, wenn es um „Andere“ geht. Da ist dann ganz selbstverständlich von „Menschen anderer Kulturen“, „Gruppen anderer Kulturen“ oder „Mitglieder der fremdkulturellen Gruppe“ die Rede, ohne dass die dahinterliegenden Konstruktionsprozesse reflektiert würden. László entfernt sich also zusehends weiter von der theoretischen Basis des Seminars. Dies zeigt sich auch in seinem Umgang mit dem Kulturbegriff im empirischen Teil. Darauf soll im nächsten Abschnitt noch genauer eingegangen werden.

\subsection{Zum Umgang mit dem Kulturbegriff im empirischen Teil der BA-Arbeit}

László hat für seine BA-Arbeit auf Ungarisch einen Fragebogen ausgearbeitet, der dann von 33 ungarischen Flugbegleiter*innen ausgefüllt wurde. Als Ziel der Arbeit gibt er an, er wolle untersuchen, ,welche Veränderungen sich in den Wahrnehmungsformen der ungarischen FlugbegleiterInnen hinsichtlich des Selbst- sowie Fremdbildes vollziehen" (V1: 24) ${ }^{11}$. Es soll nun zunächst auf die Konzeption des Fragebogens und auf den Umgang mit dem Kulturbegriff darin eingegangen werden.

10 Dabei wäre zu diskutieren, inwieweit im BA-Studium überhaupt erwartet werden kann, dass Studierende die Historizität des Diskurses überblicken.

11 Die Zitate sind dem Auswertungsteil der Arbeit entnommen, der auf Deutsch gehalten ist; die Übersetzung der ungarischen Fragen aus dem Fragebogen stammt also von László selbst. 
Im Fragebogen wird - neben demographischen Daten wie Alter und Geschlecht - erhoben, wie lange die Befragten bereits bei Qatar Airways arbeiten, wie zufrieden sie mit ihrem Arbeitgeber sind und welche anfänglichen Schwierigkeiten zu bewältigen waren. László geht mit dem Tool Fragebogen durchaus reflektiert um, nützt die Möglichkeit verschiedener Antwortformate und lässt - vereinzelt (gegen Ende) - auch offene Antworten zu.

Dem Thema Stereotype und Vorurteile sind 12 Fragen gewidmet, die teilweise auch die Interaktion mit anderen einbeziehen. Bei der Operationalisierung der Fragen zeigen sich einige Probleme im Hinblick auf die Validität einzelner Fragen: Zum Teil werden Voraussetzungen hineingetragen, die die Antworten verfälschen können. Dies ist zu einem Gutteil auf Probleme mit dem Kulturbegriff zurückzuführen. Darauf wird nun im Folgenden noch genauer eingegangen.

In Frage 1 möchte László von den Befragten wissen, worauf ihrer Ansicht nach Stereotype und Vorurteile basieren. Er gibt 8 Antwortmöglichkeiten vor: „,saját tapasztalat alapján / mások tapasztalataiból / média befolyása / történelmi esemény / kultúra, mint a szocializációs folyamat kiindulópontja / politikai hatás / neveltetés / információ hiányossága“" (V1: 38) [auf der Basis von eigenen Erfahrungen / auf Erfahrungen anderer / Einfluss der Medien / geschichtliches Ereignis / Kultur, als Ausgangspunkt der Sozialisation / politischer Einfluss / Erziehung / Informationsmangel; Übersetzung: SD]. Mehrfachantworten sind zugelassen, eine zusätzliche oder alternative offene Antwort ist aber nicht möglich. Es fällt auf, dass László hier einen Versuch unternimmt, „Kultur“ zu definieren, nämlich als „Ausgangspunkt der Sozialisation“. Hier wird auf der inhaltlichen Ebene klar, dass er sich auf einen weit gefassten Kulturbegriff bezieht, der Alltagserfahrungen mit einbezieht. Auf der extensionalen Ebene bleibt die Definition unklar, es liegt allerdings eine Interpretation in Richtung eines ethnisch verstandenen Kulturbegriffs nahe.

Besonders problematisch im Hinblick auf ihre Validität ist die Frage 2, bei der die Befragten unter vier Personen eine aussuchen sollen, die sie sympathisch finden. Die Gesichter der Personen werden nur in Ausschnitten gezeigt: $\mathrm{Zu}$ sehen ist die rechte Gesichtshälfte eines Mannes mit dunkler Hautfarbe, der mittlere Ausschnitt des Gesichts einer jungen, weißen Frau sowie eines asiatischen, sehr jung wirkenden Mannes und der linke Ausschnitt vom Gesicht eines Mannes mit kurzem Bart, Brille und einer Kufiya als Kopfbedeckung. Alle vier Menschen lächeln freundlich in die Kamera, wobei die beiden Männer rechts und links außen etwas hinter den beiden mittleren Personen, versteckt" wirken. Die dargestellten Personen unterscheiden sich im Hinblick auf ihre Hautfarbe und ihr Geschlecht. Im Falle des Mannes mit der Kufiya wurde auch auf Accessoires zurückgegriffen, die ihn einem bestimmten „Kulturkreis“ (bzw. einer bestimmten Religion?) zugehörig erscheinen lassen sollen. Es findet hier ganz offensichtlich eine Amalgamierung von Hautfarbe, Religion und Kultur statt. ${ }^{12}$

László verwendet das Bild und seine Frage dazu als eine Art Provokation und kommentiert in seiner Arbeit: „Um die stereotypische Sichtweise der befragten Stewardessen und ihre Einstellung zu anderen Menschen genau veranschaulichen zu können, mussten sie aus den folgenden Personen eine auswählen, die sie für sympathisch hielten." (V1:27) Es geht László also nicht darum, herauszufinden, inwiefern die befragten Flugbegleiter*innen mit Stereotypen und Vorurteilen operieren, sondern er möchte hier seine Vorannahme bestätigt sehen, dass sie es tun. Diese Rechnung geht auch - scheinbar - auf: 75,8 \% entscheiden sich für die (weiße) Frau. Die Art der Fragestellung legt bis zu einem gewissen Grad eine self-fulfilling prophecy nahe. Auch eventuelle Auswirkungen des Faktors Gender (75,8 \% der Befragten sind weiblich) werden nicht berücksichtigt. Das Problem wird dadurch verschärft, dass keine Mehrfachantworten (und auch keine Kommentare) möglich sind. Die Option, mehrere oder sogar alle (oder überhaupt keine) der abgebildeten Personen sympathisch zu finden, ist nicht vorgesehen - die Befragten müssen sich für eine Person entscheiden.

12 Das Bild ist eine Illustration von einer Website, die unter dem Titel „Understanding cultures“ einige ,Benimmtipps“ gibt, wie man sich in unterschiedlichen „Kulturen“ zu verhalten habe; http://lediaaesar.blogspot.hu/2015/06/understanding-cultures-have-you-ever.html [30.03.2018] 
In weiteren Fragen sollen die Flugbegleiter*innen auf einer 5-stufigen Skala ankreuzen, inwieweit sie positiv oder negativ „,zu einer anderen Kultur“ (,egy másik kultúrához“) stehen. Worauf sich der Kulturbegriff hier bezieht (die Firmenkultur bei Qatar Airways, den kulturellen Hintergrund der Passagiere oder ganz andere Aspekte) bleibt hier offen, in jedem Fall wird allerdings eine Form des Othering vorweggenommen und vorausgesetzt. Dies ist besonders interessant im Zusammenhang mit der nächsten Frage 4, in der es darum geht, $o b$ die Flugbegleiter*innen die Kategorisierung der Passagiere nach Kulturen (,kultúrák szerinti kategorizálást“; V1: 39) für wichtig halten (ja/nein). Der überwiegende Teil der Befragten (57,6 \%) antwortet hier mit „nein“, hält die Kategorisierung für nicht wichtig (V1: 25). In dieser Frage tut sich ein Fenster auf für eine potentielle Falsifizierung der Grundhypothese, dass die befragten Flugbegleiter*innen mit einer „stereotypischen Sichtweise“ an die Interaktion mit „Anderen“ herangehen. László geht auf dieses Ergebnis aber nicht weiter ein, sondern geht kommentarlos zur nächsten Frage über, ohne das Reflexionspotential zu nützen, das in diesem Ergebnis liegt.

Im Übergang zur nächsten Frage 5 ergibt sich dabei ein logischer Bruch in der Auswertung. In Frage 5 sollen die Befragten nämlich angeben, nach welchen Kriterien sie ihre Fluggäste kategorisieren. László konnte bei der Erstellung des Fragebogens natürlich noch nicht ahnen, dass die Befragten diese Kategorisierungen eigentlich gar nicht so wichtig nehmen; und dass sie sie nicht wichtig nehmen, bedeutet genau genommen auch noch nicht zwingend, dass nicht doch (unterschwellig) Kategorien gebildet werden. Ob dies der Fall ist oder nicht, wird in der Arbeit aber nicht reflektiert, sondern es wird vielmehr vorausgesetzt, dass die Flugbegleiter*innen die Fluggäste in Kategorien einteilen. Die hohe „Nein-Quote“ bei der Frage 4 hätte László eventuell bewusst machen können, dass er von Vorannahmen ausgegangen ist, die sich nicht oder nur teilweise erfüllt haben - was den Wert einer Umfrage ja keineswegs schmälern muss, im Gegenteil (vgl. Dengscherz 2016: 115ff). Es ist denkbar, dass László der Reflexion in diesem Punkt aus dem Weg geht, weil er nicht konzeptionelle Probleme in seinem Fragebogen ,aufdecken' möchte. Somit entscheidet er sich eher für das ,Zudecken' und referiert gleich im Anschluss die Ergebnisse auf die Frage 5. Die Zuordnung bezieht sich hier nun explizit auf,Volksgemeinschaften“ (,népcsoport" V1: 39). Zur Auswahl bei den Kategorien stehen Hautfarbe, Kleidung, Aussprache des Englischen, Verhalten, physische Erscheinung und hier gibt es auch zusätzlich eine offene Antwortmöglichkeit.

Die Frage 6 bezieht sich auf die Herkunft der Passagiere, es wird gefragt, aus welchen Weltregionen sie stammen. Interessant ist der Wortlaut der Frage: „Vélemenye szerint az utazók többsége melyik kultúrából származik?“(V1: 39) [Aus welcher Kultur stammt Ihrer Ansicht nach die Mehrheit der Reisenden? Übersetzung: SD]. Erst aus den Antwortmöglichkeiten (Nordamerika, Südamerika, Europa, Afrika, Mittlerer Osten, Asien, Ozeanien) wird klar, dass es sich eigentlich um geographische Regionen handelt. Es werden hier also ganz deutlich geographische und kulturelle Herkunft gleichgesetzt.

Auch in die Fragen 7 und 8 hat László Vorannahmen hineingetragen. Hier werden nun Vorurteile geradezu evoziert. In Frage 7 geht es darum, welche ethnischen Gruppen (,népcsoport“) von den Flugbegleiter*innen als besonders ,negativ“" wahrgenommen werden und in Frage 8 sollen sie angeben, welche negativen Eigenschaften sie mit ihnen verbinden. Die Fragen sind als Pflichtfragen markiert, und es werden entsprechende Stereotype (re)produziert. In Frage 9 möchte László wissen, auf welchen Faktoren die jeweilige negative Einschätzung beruht. Als Antwortmöglichkeiten sind angegeben: Kulturelle Unterschiede, mangelnde Sprachkenntnisse, Kleidung, Hautfarbe und soziale Rollen sowie eine offene Antwortmöglichkeit „anderes“ (,egyéb“) (V1: 40). In der V1 erfährt man nicht viel über die Ergebnisse, in der Endversion stellt sich heraus, dass $84,8 \%$ die - im Übrigen nicht weiter definierten - kulturellen Unterschiede für eine negative Einschätzung verantwortlich machen (EV: 27). Auch hier geht es László offenbar wieder darum, Stereotype nachzuweisen. Verzerrend wirkt, dass auch diese Fragestellung kaum zulässt, dass jemand nicht mit Stereotypen operiert. 
Der pejorative Fokus wird in der nächsten Frage 10 wieder etwas gemildert, indem László nach „positiven Überraschungen“ (,pozitív csalodás“) im Umgang „,mit Reisenden aus anderen Kulturen" („más kultúrából származó utasokkal“) fragt. Hier kommt es allerdings zu einer lexikalischen Verdrehung bei der Auswertung: László berichtet, dass 90,9\% der Befragten von positiven Erlebnissen berichten ,und nur 9,1 \% (drei Personen) Negatives erlebt haben“(V1: 29). Genau genommen hat er dies aber gar nicht erfragt: In der Frage 10 des Fragebogens geht es nicht um positive oder negative Erlebnisse an sich, sondern um positive Überraschungen ( $\mathrm{ja} / \mathrm{nein}$ ). Dieser ,Überraschungseffekt“ („,csalodás“) wird in der Auswertung nicht thematisiert (auch in der Endfassung nicht: EV: 27).

In der Frage 11 kommt László schließlich auf das zu sprechen, um das es ihm nach eigenen Angaben wesentlich in der Arbeit geht: nämlich inwiefern eine gelungene Interaktion die Einstellung zu bestimmten ethnischen Gruppen beeinflusst. Die Befragten sollen auf einer Skala von 1 bis 5 angeben, wie stark dieser Einfluss ist. (Dass er nur positiv sein kann, wird vorausgesetzt. $97 \%$ der Antworten verteilen sich auf die Skalenniveaus 3 bis 5). Die abschließende Frage 12 ist wiederum eine offene Pflichtfrage. Hier fragt László explizit nach dem Einfluss der Arbeit als Flugbegleiter*in auf Selbst- und Fremdbild(er). Bei der Auswertung der Antworten kommt László zum Schluss, dass - „,dank der multikulturellen Atmosphäre“ - die Arbeit bei Qatar Airways bei der Mehrheit der Befragten zu einer weltoffenen Auffassung und zur Öffnung zu anderen Menschen" beigetragen hat (V1: 29). László hat also keineswegs beabsichtigt, Stereotype oder Vorurteile zu reproduzieren - im Gegenteil: Er argumentiert vielmehr, dass „,multikulturelle“ Begegnungen dazu führen, dass Vorurteile abgebaut werden. Hier zeigt sich besonders deutlich, dass er von ähnlichen Voraussetzungen ausgeht, wie dies interkulturelle Trainings tun. Eine Problematisierung des homogenisierend-ethnisierenden Kulturbegriffs hätte seine Argumentationsstruktur ein gutes Stück komplexer gemacht - aber auch zusätzliches Erkenntnispotential geboten.

Nach dem Zwischenfeedback auf die V1 hat László seine Arbeit noch einmal gründlich überarbeitet. Dabei hat er sich vor allem auf die allgemeine Reflexion der Methode Fragebogen und die Darstellung der Umfrageergebnisse konzentriert und in diesen Punkten die Arbeit entscheidend verbessert. Eine tiefergehende Auseinandersetzung mit dem Kulturbegriff bleibt bei der Überarbeitung aber aus. Der theoretische Teil der Arbeit wurde etwas gekürzt, nicht zuletzt um ,Platz für die Darstellung der Forschungsmethode und der empirischen Ergebnisse zu schaffen.

\section{Diskussion der Ergebnisse}

László ist es gelungen, selbstständig eine Umfrage im Bereich inter-/multi-/transkultureller Business-Kommunikation durchzuführen: Er hat einen Fragebogen entwickelt, der von 33 ungarischen Flugbegleiter*innen bei Qatar Airways ausgefüllt wurde. Diese Einschränkung auf eine ganz bestimmte Zielgruppe und der durchaus beachtliche Rücklauf bei den Fragebögen zeigt einerseits, dass László imstande ist, ein Thema sinnvoll einzuschränken und andererseits, dass er in seiner empirischen Studie erfolgreich pragmatische Faktoren berücksichtigt hat. László beschäftigt sich mit Interaktion und bringt diese in Verbindung mit dem Abbau von Stereotypen und Vorurteilen. Dafür wollte er offenbar die Existenz von solchen Stereotypen und Vorurteilen zunächst einmal nachweisen - worauf dann ein großer Teil des Fragebogens abzielt.

Die Probleme ergeben sich nun bei der konkreten Operationalisierung. Es wird wenig präzisiert und viel in die Fragen hineingetragen, als selbstverständlich gesetzt, wo es günstiger gewesen wäre, etwas, voraussetzungsloser' zu fragen. Es zeigen sich hier zwei Grundprobleme von Fragebögen: einerseits die Herausforderung, für jede Position eine Antwortmöglichkeit zu bieten, und andererseits Schwierigkeiten im Umgang mit Indexikalität, also mit (angenommenem?) implizitem gemeinsamem Wissen (vgl. Dengscherz 2016: 124). Letzteres zeigt sich vor allem im Umgang mit dem Kulturbegriff. Hier hat László die für die Translationswissenschaft wichtigen Turns nicht mitgemacht. Das Verharren bei einem ethnisierend-homogenisierenden Kulturbegriff schafft nicht nur im theoretischen Teil Verkürzungen, sondern wirkt sich auch wesentlich auf die empirische Untersuchung aus, vor allem, indem in der Umfrage von scheinbaren Selbstverständ- 
lichkeiten ausgegangen wird, die dann die Ergebnisse verzerren. Hypothesen werden unter diesen Voraussetzungen nicht getestet, sondern es wird vor allem versucht, sie zu verifizieren - eine Falsifizierung wird durch die Art der Fragestellung erschwert, obwohl die empirischen Daten eine Falsifizierung sogar nahelegen würden.

László hat die Arbeit in seiner L2 Deutsch verfasst, die er sehr gut beherrscht. Sein Sprachgebrauch in der Arbeit entspricht weitgehend den Konventionen wissenschaftlichen Arbeitens, die Brüche, die im Text auszumachen sind, finden sich weniger auf der rhetorischen, als vielmehr auf der heuristischen Ebene. Lászlós Vorwissen über ,Kultur` steht offenbar in einem gewissen Widerspruch zu den differenzierteren Kulturkonzepten, die in den Fachtexten im Seminar Transkulturelle Kommunikation zu lesen waren (und die auch in anderen Lehrveranstaltungen im BA-Studium behandelt werden). Durch einen solchen Widerspruch ergibt sich ein Problem im Sinne von Aebli (1994: 24f.). Wenn neues Wissen als Stimulus verstanden wird, der verarbeitet werden muss, kann diese Verarbeitung nach Piaget entweder durch Assimilation oder durch Akkomodation geschehen (vgl. Piaget 2003: 53-56). Diese Prozesse lassen sich auch auf heuristisches, Wissen schaffendes Schreiben übertragen. Ortner (2000: 70) unterscheidet dabei zwischen ,schemaeinpassender Assimilation“ und ,schemaanpassender Akkomodation“. Die Akkomodation erfordert eine Veränderung der bestehenden Text- oder Wissensstruktur, wenn neue Informationen nicht in die bestehenden Schemata passen. Der Widerspruch zwischen den Schemata von Lászlós Vorwissen und den differenzierteren Kulturkonzepten, denen er im Studium begegnet, machen eine Akkomodation nötig, um den Widerspruch aufzulösen, und das Äquilibre wiederherzustellen. $\mathrm{Zu}$ einer solchen Akkomodation ist es aber nicht gekommen, sondern vielmehr zu einem Assimilationsversuch, der dazu geführt hat, dass László eine Stelle aus Saal (2014) zitiert, die zwar Saals Gesamtaussage widerspricht, in Lászlós Wissensstruktur aber passt. Damit ist das Problem - scheinbar, an der Textoberfläche - gelöst. Es zeigt sich hier ein Phänomen, das in der Entwicklung wissenschaftlicher Schreibkompetenz immer wieder zu beobachten ist: Die Schwierigkeit, mit Komplexität umzugehen (vgl. Ortner 2006: 93) und die damit einhergehende Tendenz, diese Komplexität zu reduzieren - nur nicht immer mit geeigneten Mitteln.

Interessant ist, dass das Problem keine deutlichen Spuren im Hinblick auf Inkonsistenzen in der (sprachlichen) Textgestaltung hinterlässt. Nach den von Steinhoff (2007) beschriebenen Entwicklungsphasen wäre Lászlós BA-Arbeit in Bezug auf die sprachliche Gestaltung in einem Übergangsstadium zwischen Transformation und kontextueller Passung (Steinhoff 2007: 146149) anzusiedeln. Dass er die Konventionen wissenschaftlichen Schreibens gut beherrscht und in seinem Text produzieren kann, kann auch mit seiner Studienrichtung zusammenhängen. Ein wesentlicher Studieninhalt ist es, sich Textsortenkonventionen zu erarbeiten. Dementsprechend ist denkbar, dass Studierende der Translationswissenschaft das Stadium der kontextuellen Passung auf der sprachlichen Ebene und im Umgang mit Textsortenkonventionen früher erreichen als Studierende anderer Studienrichtungen, weil sie mit entsprechend professionellen Strategien der Textanalyse und Textproduktion an die Sache herangehen.

László hat in seine Arbeit zwei seiner Arbeitssprachen produktiv einbezogen: Die Arbeit hat er auf Deutsch geschrieben und die Umfrage in seiner L1 Ungarisch durchgeführt. Bei der Übersetzung aus dem Ungarischen ins Deutsche kommt es stellenweise zu kleineren Ungenauigkeiten, die die Bedeutung von manchen Fragen etwas verzerren. Auch hier wurde manchmal etwas an Bedeutung hineininterpretiert, was in der ursprünglichen Formulierung gar nicht vorhanden war. Die stärkste Verkürzung und Ungenauigkeit ergibt sich aber nicht durch Übersetzungsprobleme, sondern im Hinblick auf den Kulturbegriff selbst. Dies manifestiert sich jedoch nicht in Problemen auf der makrostrukturellen Gestaltung: Nach den Entfaltungsniveaus von Pohl (2007: 396) wäre man geneigt, Lászlós Arbeit zwischen dem 2. und 3. (= höchsten) Entfaltungsniveau anzusiedeln: Argumentative Passagen in Lászlós Arbeit sind teils positionsbezogen, teils argumentbezogen und die Arbeit verfügt über einen konklusiven Schlussteil. In einem Punkt bleibt die Arbeit allerdings auf dem 1. Entfaltungsniveau, nämlich in Bezug auf den argumentativen Dissens. Dieser wird - in Bezug auf den Kulturbegriff - erst gar nicht eröffnet. Im theoretischen Teil wird 
nur referiert, was den empirischen (scheinbar) stützt. Dies stützt zwar auf den ersten Blick die Argumentation, allerdings um den Preis einer inhaltlichen Komplexitätsreduktion bis hin zur Verzerrung.

Es zeigt sich hier einerseits, dass in ein- und derselben Arbeit auf verschiedenen Ebenen unterschiedliche Entfaltungsniveaus zu beobachten sein können (vgl. Schindler 2017: 111), und andererseits, dass die Komplexitätsreduktion im Hinblick auf die zentralen Begriffe der Arbeit (Kultur, Identität, Stereotype, Vorurteile) die Gesamtargumentation der Arbeit entscheidend beeinträchtigt. Ein konklusiver Schlussteil ist zwar vorhanden, die Konklusion aber nur schwach aus der empirischen Studie ableitbar.

Die Probleme sind zu einem guten Teil auf Verkürzungen und Vereinfachungen des Diskurses über Kultur zurückzuführen, Knowledge Transforming - durch Input im Seminar (oder auch in anderen Lehrveranstaltungen) - hat in dieser Frage kaum stattgefunden. Eine Problematisierung des homogenisierend-ethnisierenden Kulturbegriffs wird vermieden - László bleibt im Hinblick auf den Umgang mit „Kultur“ auf dem Level der Assemblage (Yancey et al. 2014: 104), auf dem vorhandenes Wissen nur begrenzt mit neuem angereichert wird und schafft es nicht, zu einem Remix oder gar der Konstruktion einer neuen Wissensbasis zu kommen, bei dem das Vorhandene mit dem Neuen integrativ verarbeitet würde. Auch das Feedback zur Arbeit und die Möglichkeit des Überarbeitens der Arbeit haben in diesem Punkt nicht zu einem ,setback“ oder ,,criticial incident" (Yancey et al. 2014: 104) geführt - was daran liegen könnte, dass László damit beschäftigt war, die Darstellung seiner Forschungsmethode und Ergebnisse in den Griff zu bekommen (was ihm auch gelungen ist). Daneben blieb möglicherweise nicht mehr ausreichend Zeit, Energie (und Motivation), um sich auch noch einmal tiefergehend mit Kulturkonzepten auseinanderzusetzen.

Eine tiefergehende Reflexion des Kulturbegriffs hätte außerdem bedeutet, sich mit dem eigenen Fragebogen kritisch auseinandersetzen zu müssen und eventuelle Brüche oder Stolpersteine in der empirischen Untersuchung aufzuzeigen. Der Fragebogen selbst konnte zu diesem Zeitpunkt allerdings nicht mehr geändert werden. Eine nachträgliche kritische Reflexion ohne Überarbeitungsmöglichkeit könnte deshalb als eine ,Analyse des Scheiterns' aufgefasst werden, für die nur schwer Motivation aufzubringen ist. ${ }^{13}$ Hier ergeben sich sinnvolle Anknüpfungspunkte für weiterführende Forschung.

\section{Schlussfolgerungen}

Es lässt sich insgesamt fragen, inwieweit von BA-Studierenden verlangt werden kann, was selbst im Fachdiskurs nicht immer erfüllt wird - Verkürzungen im Umgang mit dem Kulturbegriff sind ein weit verbreitetes Phänomen: So hat etwa Diehm (2010: 79) festgestellt, dass „,begriffliche Verengungen, wie das Ineinssetzen von Nationalität und Kultur oder von Kultur und Religion dann trotz anderslautenden Behauptungen und Intentionen offenbar doch nicht so einfach zu vermeiden“ sind, „so als wären sie letztendlich nicht mehr aus den Köpfen zurückzuholen“. Das gilt keineswegs nur für Studierende. Saal (2014: 33ff) und Hormel/Jording (2016: 219) weisen darauf hin, dass selbst Welsch in seinem Transkulturalitätskonzept (vgl. z.B. Welsch 1997, 2010) diese Überwindung nicht völlig gelungen ist. Der holistisch-ethnologische Kulturbegriff und ein differenzierungstheoretisches Kulturverständnis liegen also immer noch im Widerstreit (vgl. Darows$\mathrm{ka} /$ Machold 2010: 18).

Diese Phänomene zeigen sich auch in den Lernprozessen im Studium der Transkulturellen Kommunikation: Transkulturalität, Kulturalismus, Othering, Third Space etc. sind Begriffe, die Studierenden immer wieder begegnen und die sie teilweise auch schlagwortartig übernehmen. Diese Begriffe mit Konzepten zu füllen und diese Konzepte zu verstehen und selbstständig anzuwenden, gelingt nicht immer sofort - und auch die Motivation dafür ist nicht bei allen Studie-

13 Dazu kommt, dass Stolpersteine im Forschungsprozess auch in Fachtexten nur recht selten reflektiert werden (vgl. Kruse 2015: 635; Dengscherz 2016: 116), wodurch sich Studierende auch kaum an entsprechenden Vorbildern in der Fachliteratur orientieren können. 
renden gleichermaßen vorhanden. Die Skepsis gegenüber dem homogenisierend-ethnisierenden Kulturbegriff wird zuweilen als vor allem ideologisch motiviert, wenn nicht sogar praxisfern wahrgenommen. Hier gilt es m.E. didaktisch anzusetzen und zu zeigen, wie durch ein differenzierteres Kulturverständnis auch ein differenzierterer Blick auf den Gegenstand möglich wird was nicht nur zu einem vertieften theoretischen Verständnis führt, sondern auch zu effizienterem translatorischem Handeln.

Es stellt sich die Frage, was es - (translations-)didaktisch - braucht, um ein solches differenzierteres Kulturverständnis zu befördern. Ist der Begriff „Kultur“ an sich zu problematisieren? Diehm (2010) äußert (im Kontext der Erziehungswissenschaft) eine so weitgehende Skepsis gegenüber dem Kulturbegriff, dass sie vorschlägt, stattdessen

\begin{abstract}
auf ein anderes programmatisches und konzeptuelles Gleis zu setzen. Sinnvoller und angemessener als der Fokus auf Kultur vermag die Perspektive auf Differenz in einem generellen Sinne den Horizont der Anforderungen umreißen, der den sozialen Umgang in modernen Gesellschaften ausmacht. (Diehm 2010: 79)
\end{abstract}

Hier wäre zunächst einmal zu klären, in welchen Dimensionen diese Differenzlinien verlaufen und die Dimensionen wären zu benennen. Es wäre zu überlegen, ob nicht in vielen Fällen, dort, wo der Begriff „Kultur“ verwendet wird, eigentlich etwas anderes gemeint ist - und ob es nicht dementsprechend eine präzisere Bezeichnung für das Gemeinte gibt: Gesellschaft, Markt, Region, Religion, Domäne, Interessensgemeinschaft, Diskursgemeinschaft oder auch Firmenkultur, Institutionskultur etc. Das Ziel dabei kann nicht sein, den Begriff „Kultur“ völlig zu vermeiden oder auf das zu reduzieren, was nach all den ,Umbenennungen' übrig bleibt - nach dem Motto: ,Und der Rest ist Kultur'. Sicherlich wäre es aber von Vorteil, den in manchen Texten inflationären und häufig sehr schwammigen Gebrauch des Begriffs zu hinterfragen, gezielt einzuschränken und auf diese Weise für mehr Präzision zu sorgen. Über inhaltliche und lexikalische Präzisierungen und letztendlich Ersetzungen des Kulturbegriffs Klarheit über seine unterschiedlichen Bedeutungsebenen zu erlangen, kann eine sehr gute Übung für Studierende sein, um sich über konzeptuelle Probleme des Kulturbegriffs klar zu werden. Im Falle von Lászlós BA-Arbeit wäre eine solche Präzisierungsarbeit schon für die Operationalisierung des Kulturbegriffs im Fragebogen von Vorteil gewesen - hätte aber auch abgesehen davon bei der Reflexion der Auswertung geholfen.

Darüber hinaus wäre wünschenswert, dass ,,setbacks“ und „critical incidents“ (Yancey et al. 2014: 104) in der Auseinandersetzung mit „Kultur“ und den dahinterliegenden Konzepten explizit als Lerngelegenheiten wahrgenommen werden (können) und so langfristig zu einer Vertiefung der Reflexion führen. Möglicherweise hätte eine solche Vertiefung der Reflexion auch in Bezug auf den Kulturbegriff und seine Operationalisierung im Fragebogen stattfinden können, wenn die Semestereinteilung noch einen zweiten Überarbeitungsdurchgang nach nochmaligem Feedback zugelassen hätte - bei dem sich László dann speziell auf diesen einen Aspekt hätte konzentrieren können. Für die Vertiefung eines solchen Themas im Rahmen einer empirischen Studie kann ein Semester durchaus zu knapp werden.

Didaktisch braucht es sicherlich noch weitere Bemühungen, die auf die Bereitschaft der Studierenden abzielen, sich auf konkurrierende Konzepte und argumentativen Dissens einzulassen. Es geht darum, dass sie erfahren, dass Spannungen sehr produktiv sein können, wie Canagarajah (2001) in einer autobiographischen Notiz über sein eigenes Schreiben in unterschiedlichen Diskursgemeinschaften in Sri Lanka und den USA ausführt, in dem er feststellt: "being caught between conflicting and competing writing traditions, discourses, or languages is not always a 'problem'. These tensions can be resourceful in enabling a rich repertoire of communicative strategies.” (2001: 36) Im Idealfall führen diese Spannungen zu ,useful insights into the ideological and rhetorical challenges in academic communication" (S. 37). Canagarajah (2001) kommt übrigens in seiner Darstellung der eigenen Multiliteralität ganz ohne den Begriff ,culture' aus - sondern verwendet statt dessen analytisch konkrete Subbegriffe, die jeweils den Aspekt präzisieren, der gemeint ist (writing traditions, discourses, languages, academic communication). 


\section{Ausblick}

Durch den „,cultural turn“ wurden Konzepte und theoretische Ansätze ausgeweitet, die die Homogenisierung und Ethnisierung des Kulturbegriffs in Frage stellen und stattdessen „Aspekte der Grenzüberschreitung und Grenzüberschneidung sowie den Prozesscharakter und die Dynamik von Kultur" betonen (vgl. Saal 2014: 21). Diese Konzepte und Ansätze sind im Fachdiskurs bereits weit verbreitet, Studierende haben aber teilweise recht große Schwierigkeiten, sie nachzuvollziehen und mit ihnen zu arbeiten. Die Analyse hat dies exemplarisch anhand einer BA-Arbeit aus dem Bereich der transkulturellen Business-Kommunikation demonstriert.

Die Ausdifferenzierung des Kulturbegriffs, auch in seiner extensionalen Dimension ist ein wesentlicher Aspekt im Diskurs zur Transkulturellen Kommunikation. Welches Potential in einer solchen Ausdifferenzierung liegt - und welches Potential verschenkt wird, wenn sie ausbleibt lässt sich besonders gut anhand von konkreten Beispielen (wie eben Arbeiten von Studierenden) zeigen. Ein Ziel der vorliegenden Fallstudie ist es gewesen, dies nachvollziehbar zu machen.

Für die Translationsdidaktik ergibt sich daraus die durchaus wesentliche Frage, wie Studierende dabei unterstützt werden können, sich - schreibend - mit Kulturkonzepten auseinanderzusetzen, die Hybridisierung und dynamische Veränderungen mit reflektieren und dadurch der Gefahr der Ethnisierung, Homogenisierung und Essentialisierung entgehen, indem sie präzisiere Gegenstandsdefinitionen vornehmen. Dabei ist es sinnvoll, die heuristischen und rhetorischen Anforderungen und Herausforderungen (vgl. Dengscherz 2017, 2018) näher zu beleuchten, die sich beim Schreiben über „Kultur“ ergeben und ihre Auswirkungen auf den Schreibprozess zu analysieren: Auf der heuristischen Ebene lässt sich fragen, wie differenzierte Konzeptualisierungen von „Kultur" auf unterschiedliche Forschungsfragen im Feld der Transkulturellen Kommunikation angewendet werden können und wie sich aus dieser Anwendung auch differenzierteres Erkenntnispotential ergibt. Auf der rhetorischen Ebene kann es wiederum um die Frage gehen, wie Konzepte jeweils präzisiert werden können und wo tatsächlich „Kultur“ gemeint ist und wo hingegen ein anderer Begriff klarer und eindeutiger wäre. Diese Fragen wurden im vorliegenden Beitrag exemplarisch an einer BA-Arbeit untersucht.

Aus der Analyse ergeben sich bereits einige didaktische Anknüpfungspunkte, es braucht aber sicherlich noch weitere Studien dazu, um eine breitere Basis schaffen, auf der didaktisch aufgebaut werden kann. Somit ergeben sich Desiderate für weitere Forschung, sowohl produktorientiert als auch prozessorientiert. Auf der Produktebene wäre es sinnvoll, das Phänomen in weiteren studentischen Arbeiten zu untersuchen, auch im Hinblick auf die Frage, ob und wie sich eventuelle Übergangsphänomene - wie die teilweise Verarbeitung differenzierterer Konzepte von „Kultur" - in studentischen Arbeiten äußern. Auf der Prozessebene wäre es aufschlussreich, herauszufinden, inwieweit heuristische und/oder rhetorische Herausforderungen im Schreibprozess sichtbar werden und welche Entscheidungen die Studierenden bei auftauchenden Problemen treffen. Hier könnte etwa der Einsatz von Integrated Problem Decision Reports (IPDR, vgl. Heine 2012) sowie Keystroke-Logging (vgl. Leijten/Van Waes 2013) und/oder Screen-Capturing-Methoden (vgl. Dengscherz 2017, 2018) in Kombination mit Interview-Techniken wie dem StrukturlegeVerfahren oder dem Dialog-Konsensus-Interview (vgl. Heine et al. 2014) wertvolle Daten liefern.

Auf Basis eines konkreten heuristischen Problems (und seiner rhetorischen Verarbeitung) lässt sich erforschen, wie Schreibende individuell unterschiedlich Lösungen ausarbeiten. So könnten mehrere Studierende unabhängig voneinander an der gleichen Schreibaufgabe arbeiten und ihre Schreibprozesse dokumentieren und analysieren (mit den oben genannten Methoden). Die vorliegende Analyse und die vorgeschlagenen weiterführenden Erhebungsmethoden bieten der Translationsdidaktik wertvolles Material, dass dazu genützt werden kann, Studierende beim Schreiben über „Kultur“ zu unterstützen. Ziel ist, sie davor zu bewahren, durch einen undifferenzierten Umgang mit dem Kulturbegriff ihre theoretische und empirische Arbeit auf Vorannahmen aufzubauen, die den Erkenntnisprozess nachhaltig behindern. Es gilt, Wege aufzuzeigen, wie ein differenzierteres Kulturverständnis zu einem präziseren und vertieften Verständnis (empirischer) Studien 
führen kann, das sich auch fruchtbar für translatorisches Handeln erweist. Der vorliegende Beitrag hat exemplarisch gezeigt, wie Analyseschritte auf solchen Wegen aussehen können.

\section{Literatur}

Aebli, Hans 1994: Denken: Das Ordnen des Tuns. Band II: Denkprozesse. Stuttgart: Klett-Cotta.

Bathia, Vijay K. 2015: Critical Genre Analysis: Theoretical Preliminaries. In Hermes - Journal of Language and Communication in Business no 54, 9-20.

Bachmann-Medick, Doris 2006: Cultural Turns. Neuorientierungen in den Kulturwissenschaften. Reinbek bei Hamburg: Rowohlt.

Beaufort, Anne/Iñesta, Anna 2014: Author Profiles: Awareness, competence, and skills. In Jakobs, Eva-Maria/ Perrin, Daniel (Hrsg.), Handbook of Writing and Text Production. Berlin/Boston: De Gruyter, 141-158.

Bereiter, Carl/Scardamalia, Marlene 1987: The Psychology of Written Composition. New York/London: Routledge.

Bhabha, Homi K. 1994: The Location of Culture. London: Routledge.

Bransford, John D./Pellegrino, James W./Donovan Suzanne M. 2000: How People Learn: Brain, Mind, Experience and School. Expanded Edition. Washington, DC: National Academy Press.

Canagarajah, Suresh 2001: The Fortunate Traveler: Shuttling between Communities and Literacies by Economy Class. In Belcher, Diane/Connor, Ulla (Hrsg.), Reflections on Multiliterate Lives. Clevedon: Multilingual Matters, 23-37.

Castro Varela, María do Mar 2010: Interkulturelles Training? Eine Problematisierung. In Darowska, Lucyna/Lüttenberg, Thomas/Machold, Claudia (Hrsg.), Hochschule als transkultureller Raum? Kultur, Bildung und Differenz in der Universität. Bielefeld: Transcript Verlag, 117-129.

Darowska, Lucyna/Machold, Claudia 2010: Hochschule als transkultureller Raum unter den Bedingungen von Internationalisierung und Migration - eine Annäherung. In Darowska, Lucyna/Lüttenberg, Thomas/Machold, Claudia (Hrsg.), Hochschule als transkultureller Raum? Kultur, Bildung und Differenz in der Universität. Bielefeld: Transcript Verlag, 13-37.

Dengscherz, Sabine 2018: Heuristische und rhetorische Herausforderungen meistern - Strategien für wissenschaftliches Formulieren in der L2 Deutsch. In Nied Curcio, Martina/ Cortés Velásquez, Diego (Hrsg.), Strategien im Kontext des lebenslangen und mehrsprachigen Lernens. Berlin: Frank\&Timme, 211-232.

Dengscherz, Sabine 2017: Strategien und Routinen für wissenschaftliches Schreiben in der L2 Deutsch. Zwischenergebnisse aus dem Projekt PROSIMS. In ÖDaF-Mitteilungen 2017/1, 157-173.

Dengscherz, Sabine 2016: Mehrsprachiges Arbeiten, Kooperation und Interaktion. Teilergebnisse einer Fragebogenstudie zur Mehrsprachigkeit am Zentrum für Translationswissenschaft (ZTW) - und einige methodologische Randnotizen. In Richter, Julia/Zwischenberger, Cornelia/Kremmel, Stefanie/Spitzl, Karlheinz (Hrsg.), (Neu-)Kompositionen. Aspekte transkultureller Translationswissenschaft. Berlin: Frank\&Timme, 103-129.

Diehm, Isabell 2010: Kultur als Beobachtungsweise. In Darowska, Lucyna/Lüttenberg, Thomas/Machold, Claudia (Hrsg.), Hochschule als transkultureller Raum? Kultur, Bildung und Differenz in der Universität. Bielefeld: Transcript Verlag, 67-81.

Dizdar, Dilek 2006: Translation. Um- und Irrwege. Berlin: Frank\&Timme.

Ehlich, Konrad 1999: Alltägliche Wissenschaftssprache. In InfoDaF 26, 3-24.

Hall, Stuart 1994: Rassismus und kulturelle Identität. Hamburg: Argument.

Hansen, Klaus P. 2009: Kultur, Kollektiv, Nation. Passau: Verlag Karl Stutz.

Heine, Carmen 2012: Prozessansatz im traditionell produktorientierten ,Academic Writing 'Textproduktionsunterricht. In Knorr, Dagmar/Verhein-Jarren, Annette (Hrsg.), Schreiben unter Bedingungen von Mehrsprachigkeit. Frankfurt/ Main: Peter Lang, 2012 (=Textproduktion und Medium 12), 99-116.

Heine, Carmen/Engberg, Jan/Knorr, Dagmar/Spielmann, Daniel 2014: New methods of text production process research combined. In Knorr, Dagmar/Heine, Carmen/Engberg, Jan (eds.), Methods in writing research. Frankfurt/M.: Peter Lang, 123-145.

Hofstede, Geert/Hofstede, Gert Jan/Minkov, Michael 2010: Cultures and Organizations. Software of the Mind. Intercultural Cooperation and Its Importance for Survival. New York u.a.: McGraw Hill.

Hormel, Ulrike/Jording, Judith 2016: Kultur/Nation. In Mecheril, Paul (Hrsg.), Handbuch Migrationspädagogik. Weinheim und Basel: Beltz Verlag, 211-225.

Jäger, Siegfried (2009): Kritische Diskursanalyse. Eine Einführung. 5. Auflage. Münster: Unrast Verlag.

Jäger, Margarete/Jäger, Siegfried 2007: Deutungskämpfe. Theorie und Praxis Kritischer Diskursanalyse. Wiesbaden: VS Verlag für Sozialwissenschaften. 
Kadrić, Mira/Kaindl, Klaus/Kaiser-Cooke, Michèle 2005: Translatorische Methodik. Wien: Facultas Verlag (= Basiswissen Translation 1).

Kruse, Jan 2015: Qualitative Interviewforschung. Ein integrativer Ansatz. 2., überarbeitete und ergänzte Auflage. Weinheim und Basel: Beltz Juventa.

Kaiser-Cooke, Michèle 2007: Wissenschaft, Translation, Kommunikation. Wien: Facultas Verlag (= Basiswissen Translation 2).

Kellogg, Ronald T. 2008: Training writing skills: A cognitive developmental perspective. In Journal of Writing Research 1, 1-26.

Knappik, Magdalena 2017: Schreiben für Viabilität. Subjektivierungsprozesse von Schreibenden in der Migrationsgesellschaft. Dissertation. Universität Wien.

Knappik, Magdalena 2013: Wege zur wissenschaftlichen Textkompetenz - Schreiben für reflexive Professionalisierung. Ein förderdiagnostisches Instrument zur Unterstützung von Studierenden bei der Aneignung wissenschaftlicher Textkompetenz. Wien: bm:uk [online]. [https://bildung.bmbwf.gv.at/schulen/unterricht/ba/sprachen_sfrp_ broschuere_26215.pdf?4dzgm2, 9.4.2018].

Layes, Gabriel 2005: Kulturdimensionen. In Thomas, Alexander/Kinast, Eva-Ulrike/Schroll-Machl, Sylvia (Hrsg.), Handbuch Interkulturelle Kommunikation und Kooperation. Band 1: Grundlagen und Praxisfelder. Göttingen: Vandenhoek \& Ruprecht, 60-73.

Leijten, Mariëlle/VanWaes, Luuk 2013: Keystroke Logging in Writing Research. Using Inputlog to Analyze and Visualize Writing Processes. In Written Communication 2013/3, 358-392.

Lutter, Christina/Reisenleitner, Markus 2002: Introducing History (in)to cultural studies: some remarks on the Germanspreaking context. In Cultural Studies 16/5, 611-630.

Mae, Michiko/Saal, Britta (Hrsg.) 2014: Transkulturelle Genderforschung. Ein Studienbuch zum Verhältnis von Kultur und Geschlecht. 2. Auflage. Wiesbaden: Springer.

Mecheril, Paul/Klingler, Birte 2010: Universität als transgressive Lebensform. Anmerkungen, die gesellschaftliche Differenz- und Ungleichheitsverhältnisse berücksichtigen. In Darowska, Lucyna/Lüttenberg, Thomas/Machold, Claudia (Hrsg.), Hochschule als transkultureller Raum? Kultur, Bildung und Differenz in der Universität. Bielefeld: Transcript Verlag, 83-116.

Mecheril, Paul/Dirim, Inci (Hrsg.) 2010: Spannungsverhältnisse. Interkulturell-pädagogische Forschung, Neo-Assimilationismus und migrationsgesellschaftliche Wirklichkeit. München: Waxmann.

Meyer, Bernd 2016: Case Studies. In Angelelli, Claudia V./Baer, Brian James (eds), Researching Translation and Interpreting. New York: Routledge, 177-184.

Molitor, Sylvie 1985: Personen- und aufgabenspezifische Schreibstrategien. Fünf Fallstudien. In Unterrichtswissenschaft 1985/4, 334-345.

Ortner, Hanspeter 2006: Spontanschreiben und elaboriertes Schreiben - wenn die ursprüngliche Lösung zu einem Teil des (neuen) Problems wird. In Kissling, Walter/Perko, Gudrun (Hrsg.), Wissenschaftliches Schreiben in der Hochschullehre. Reflexionen, Desiderate, Konzepte. Innsbruck: Studienverlag, 77-101.

Ortner, Hanspeter 2000: Schreiben und Denken. Niemeyer, Tübingen (= Reihe Germanistische Linguistik 214).

Piaget, Jean 2003: Meine Theorie der geistigen Entwicklung. Weinheim und Basel: Beltz Verlag.

Pöchhacker, Franz 1994: Simultandolmetschen als komplexes Handeln. Tübingen: Narr (= Language in Performance 10).

Pohl, Thorsten 2007: Studien zur Ontogenese wissenschaftlichen Schreibens. Tübingen: Niemeyer (= Reihe Germanistische Linguistik 271).

Reiß, Katharina/Vermeer, Hans J. 1984: Grundlegung einer allgemeinen Translationstheorie. Tübingen: Niemeyer (= Linguistische Arbeiten 147).

Resch, Renate 2012: Feuerroutine? Textroutine! Textsortenkompetenz in DaF und DaZ. In ÖDaF-Mitteilungen 2012/2, 46-64.

Resch, Renate, 2006: Translatorische Textkompetenz. Texte im Kulturtransfer. Frankfurt: Peter Lang Verlag.

Resch, Renate 1999: Textproduktion. In Snell-Hornby, Mary/Hönig, Hans G./Kußmaul, Paul/Schmitt, Peter A. (Hrsg.), Handbuch Translation. 2., verbesserte Auflage, 164-167.

Risku, Hanna 1999: Translatorisches Handeln. In Snell-Hornby, Mary/Hönig, Hans G./Kußmaul, Paul/Schmitt, Peter A. (Hrsg.), Handbuch Translation. 2., verbesserte Auflage, 107-112.

Saal, Britta 2014: Kultur in Bewegung. In Mae, Michiko/Saal, Britta (Hrsg.), Transkulturelle Genderforschung. Ein Studienbuch zum Verhältnis von Kultur und Geschlecht. 2. Auflage. Wiesbaden: Springer, 21-47.

Said, Edward 1978: Orientalism. New York: Vintage Books. 
Schindler, Kirsten 2017: Studium und Beruf. In Becker-Mrotzek, Michael/Grabowski, Joachim/Steinhoff, Torsten (Hrsg.), Forschungshandbuch empirische Schreibdidaktik. München: Waxmann, 109-123.

Spivak, Gayatri Chakravorty 1988: Can the Subaltern Speak? In Nelson, Cary/Grossberg, Lawrence (eds.), Marxism and the Interpretation of Culture. Houndmills et al.: Macmillan Education LTD.

Steinhoff, Torsten 2007: Wissenschaftliche Textkompetenz. Sprachgebrauch und Schreibentwicklung in wissenschaftlichen Texten von Studenten und Experten. Tübingen: Niemeyer (= Reihe Germanistische Linguistik 280).

Thomas, Alexander 2005: Kultur und Kulturstandards. In Thomas, Alexander/Kinast, Eva-Ulrike/Schroll- Machl, Sylvia (Hrsg.), Handbuch Interkulturelle Kommunikation und Kooperation. Band 1: Grundlagen und Praxisfelder. Göttingen: Vandenhoeck \& Ruprecht, 19-32.

Thomas-Olalde, Oscar/Velho, Astride 2011: Othering and its Effects - Exploring the Concept. In Niedrig, Heike/ Ydesen, Christian (Hrsg.), Writing Postcolonial Histories of Intercultural Education. Frankfurt/Main: Peter Lang Verlag, 27-51.

Vermeer, Hans J. 2007: Linguistik und Translationswissenschaft - oder Sinn und Leistung des Kulturbegriffs in der Translation. In ders., Ausgewählte Vorträge zur Translation und anderen Themen. Selected Papers on Translation and other Subjects. Berlin: Frank\&Timme (= TransÜD Arbeiten zur Theorie und Praxis des Übersetzens und Dolmetschens), 105-135.

Welsch, Wolfgang 2010: Was ist eigentlich Transkulturalität? In Darowska, Lucyna/Lüttenberg, Thomas/Machold, Claudia (Hrsg.), Hochschule als transkultureller Raum? Kultur, Bildung und Differenz in der Universität. Bielefeld: Transcript Verlag, 39-66.

Welsch, Wolfgang 1997: Transkulturalität. Zur veränderten Verfassung heutiger Kulturen. In Schneider, Irmela/Thomsen, Christian W. (Hrsg.), Hybridkultur. Medien, Netze, Künste. Köln: Wienand Verlag, 67-90.

Witte, Heidrun 2000: Die Kulturkompetenz des Translators. Begriffliche Grundlegung und Didaktisierung. Tübingen: Stauffenburg Verlag.

Yancey, Kathleen Blake/Robertson, Liane/Taczak, Kara 2014: Writing Across Contexts. Transfer, Composition, and Sites of Writing. Boulder: University Press of Colorado.

Yin, Robert 2009. Case Study Research. Design and Methods. Thousand Oaks: SAGE (= Applied Social Research 5). 